\title{
Splitting formulas for the LMO invariant of rational homology three-spheres
}

\author{
GWÉNAËL MASSUYEAU
}

\begin{abstract}
For rational homology 3-spheres, there exist two universal finite-type invariants: the Le-Murakami-Ohtsuki invariant and the Kontsevich-Kuperberg-Thurston invariant. These invariants take values in the same space of "Jacobi diagrams", but it is not known whether they are equal. In 2004, Lescop proved that the KKT invariant satisfies some "splitting formulas" which relate the variations of KKT under replacement of embedded rational homology handlebodies by others in a "Lagrangian-preserving" way. We show that the LMO invariant satisfies exactly the same relations. The proof is based on the LMO functor, which is a generalization of the LMO invariant to the category of 3-dimensional cobordisms, and we generalize Lescop's splitting formulas to this setting.
\end{abstract}

$57 \mathrm{M} 27$

\section{Introduction}

A rational homology 3-sphere (or $\mathbb{Q}$-homology 3-sphere) is a closed oriented 3manifold $S$ that has the same homology with rational coefficients as the standard 3-sphere $S^{3}$. Le, Murakami and Ohtsuki defined in [16] an invariant $Z(S)$ of rational homology 3 -spheres $S$ with values in the algebra $\mathcal{A}(\varnothing)$ of Jacobi diagrams. The LMO invariant $Z(S)$, which was originally denoted by $\widehat{\Omega}(S)$ in [16], is multiplicative under connected sums. As shown in [4], it coincides with the Aarhus integral $\stackrel{\circ}{A}(S)$ introduced by Bar-Natan, Garoufalidis, Rozansky and Thurston [2; 3]. This paper is aimed at studying the behavior of $Z$ under a certain type of rational homology handlebody replacement, called "Lagrangian-preserving surgery" by Lescop [17] and whose definition we now recall.

A rational homology handlebody (or $\mathbb{Q}$-homology handlebody) of genus $g$ is a compact oriented 3-manifold $C^{\prime}$ that has the same homology with rational coefficients as the standard genus- $g$ handlebody. The Lagrangian of $C^{\prime}$ is the kernel $\boldsymbol{L}_{C^{\prime}}^{\mathbb{Q}}$ of the homomorphism incl $_{*}: H_{1}\left(\partial C^{\prime} ; \mathbb{Q}\right) \rightarrow H_{1}\left(C^{\prime} ; \mathbb{Q}\right)$ induced by the inclusion: indeed, this is a Lagrangian subspace of $H_{1}\left(\partial C^{\prime} ; \mathbb{Q}\right)$ with respect to the intersection pairing. A $\mathbb{Q}$-Lagrangian-preserving pair (or $\mathbb{Q}-L P$ pair) is a pair $C=\left(C^{\prime}, C^{\prime \prime}\right)$ of two rational 
homology handlebodies whose boundaries are identified $\partial C^{\prime} \equiv \partial C^{\prime \prime}$ in such a way that $\boldsymbol{L}_{C^{\prime}}^{\mathbb{Q}}=\boldsymbol{L}_{C^{\prime \prime}}^{\mathbb{Q}}$. The total manifold of the $\mathbb{Q}$-LP pair C is the closed oriented 3-manifold

$$
C:=\left(-C^{\prime}\right) \cup_{\partial} C^{\prime \prime}
$$

Note that the inclusion $C^{\prime} \subset C$ induces a canonical isomorphism $H_{1}\left(C^{\prime} ; \mathbb{Q}\right) \simeq$ $H_{1}(C ; \mathbb{Q})$. The form $H^{1}(C ; \mathbb{Q})^{\otimes 3} \rightarrow \mathbb{Q}$ defined by triple cup products $(x, y, z) \mapsto$ $\langle x \cup y \cup z,[C]\rangle$ is skew-symmetric; we denote it by

$$
\mu(C) \in \operatorname{Hom}_{\mathbb{Q}}\left(\Lambda^{3} H^{1}(C ; \mathbb{Q}), \mathbb{Q}\right) \simeq \Lambda^{3} H_{1}(C ; \mathbb{Q}) .
$$

Given a compact oriented 3-manifold $M$ and a $\mathbb{Q}$-LP pair $C=\left(C^{\prime}, C^{\prime \prime}\right)$ such that $C^{\prime}$ is embedded in the interior of $M$, one can replace the submanifold $C^{\prime}$ in $M$ by $C^{\prime \prime}$ in order to obtain a new 3-manifold

$$
M_{\mathrm{C}}:=\left(M \backslash \operatorname{int}\left(C^{\prime}\right)\right) \cup_{\partial} C^{\prime \prime} .
$$

The move $M \rightsquigarrow M_{\mathrm{C}}$ between compact oriented 3-manifolds is called a $\mathbb{Q}-L P$ surgery.

Suppose now that we are given a rational homology 3-sphere $S$ and a finite family of $\mathbb{Q}$-LP pairs $C=\left(C_{1}, \ldots, C_{r}\right)$, where $C_{i}^{\prime} \subset S$ and $C_{i}^{\prime} \cap C_{j}^{\prime}=\varnothing$ for all $i \neq j$. We associate to the family $\mathrm{C}$ the tensor

$$
\mu(\mathrm{C}):=\mu\left(C_{1}\right) \otimes \cdots \otimes \mu\left(C_{r}\right) \in \bigotimes_{i=1}^{r} \Lambda^{3} H_{1}\left(C_{i} ; \mathbb{Q}\right) \subset S^{r} \Lambda^{3} H_{1}(C ; \mathbb{Q}),
$$

where we have set $C:=C_{1} \sqcup \cdots \sqcup C_{r}$ so that $H_{1}(C ; \mathbb{Q})=H_{1}\left(C_{1} ; \mathbb{Q}\right) \oplus \cdots \oplus H_{1}\left(C_{r} ; \mathbb{Q}\right)$. Besides, the linking number in $S$ defines for any $i \neq j$ a linear map

$$
\ell_{i, j}: H_{1}\left(C_{i}^{\prime} ; \mathbb{Q}\right) \times H_{1}\left(C_{j}^{\prime} ; \mathbb{Q}\right) \longrightarrow \mathbb{Q}
$$

by setting $\ell_{i, j}([K],[L]):=\operatorname{Lk}_{S}(K, L)$ for any oriented knots $K \subset C_{i}^{\prime}$ and $L \subset C_{j}^{\prime}$; thus we can also associate to $C$ the symmetric bilinear form

$$
\ell_{S}(\mathrm{C}):=\sum_{i \neq j} \ell_{i, j}: H_{1}(C ; \mathbb{Q}) \times H_{1}(C ; \mathbb{Q}) \longrightarrow \mathbb{Q},
$$

where $H_{1}(C ; \mathbb{Q})$ is identified to $H_{1}\left(C_{1}^{\prime} ; \mathbb{Q}\right) \oplus \cdots \oplus H_{1}\left(C_{r}^{\prime} ; \mathbb{Q}\right)$ in the canonical way.

We now recall how symmetric products of antisymmetric 3-tensors such as (1-1) can be depicted graphically using Jacobi diagrams. For any $\mathbb{Q}$-vector space $V$, the space of $V$-colored Jacobi diagrams is

$$
\frac{\mathbb{Q} \cdot\left\{\begin{array}{c}
\text { finite unitrivalent graphs whose trivalent vertices are } \\
\text { oriented and whose univalent vertices are colored by } V
\end{array}\right\}}{\text { AS, IHX, multilinearity }}
$$


Here an orientation of a trivalent vertex is a cyclic ordering of the incident half-edges (which, on pictures, is given by the counterclockwise direction) and the relations are:

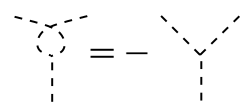

AS

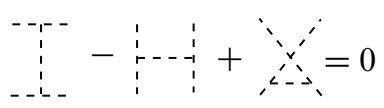

IHX

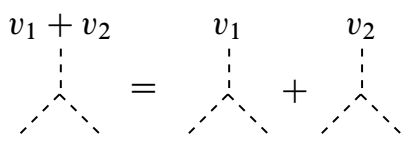

multilinearity

With the disjoint union $\sqcup$ of diagrams, $\mathcal{A}(V)$ is a commutative algebra. The internal degree (or $i$-degree) of a Jacobi diagram is the number of trivalent vertices. Then any symmetric product of antisymmetric 3-tensors

$$
\left(v_{1} \wedge w_{1} \wedge t_{1}\right) \cdots\left(v_{r} \wedge w_{r} \wedge t_{r}\right) \in S^{r} \Lambda^{3} V
$$

can be seen as the Jacobi diagram

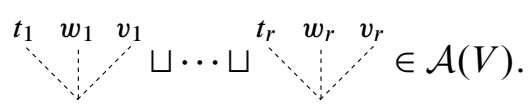

If we are given a symmetric bilinear form $\ell: V \times V \rightarrow \mathbb{Q}$, then we can produce from (1-4) a new element of $\mathcal{A}(V)$ by matching pairwisely some of its univalent vertices and by multiplying the resulting diagram with the values of $\ell$ on the corresponding pairs of vertices. We shall say that we have glued with $\ell$ some legs of (1-4). In particular, if $r$ is even, then we can glue with $\ell$ all legs of (1-4) to get an element of

$$
\mathcal{A}(\varnothing):=\mathcal{A}(\{0\})=\frac{\mathbb{Q} \cdot\{\text { finite trivalent graphs whose vertices are oriented }\}}{\text { AS, IHX }} .
$$

Note that $\mathcal{A}(\varnothing)$ is the algebra where the LMO invariant $Z$ of rational homology 3 -spheres takes values. The above terminology being fixed, we can now state our main result.

Theorem Let $S$ be a rational homology 3-sphere and let $\mathrm{C}=\left(\mathrm{C}_{1}, \ldots, \mathrm{C}_{r}\right)$ be a finite family of $\mathbb{Q}-L P$ pairs such that $C_{i}^{\prime} \subset S$ and $C_{i}^{\prime} \cap C_{j}^{\prime}=\varnothing$ for all $i \neq j$. For any $I \subset\{1, \ldots, r\}$, we denote by $S_{C_{I}}$ the manifold obtained from $S$ by the $\mathbb{Q}-L P$ surgeries $S \rightsquigarrow S_{\mathrm{C}_{i}}$ performed simultaneously for all $i \in I$. Then we have the following "splitting formula",

$$
\sum_{I \subset\{1, \ldots, r\}}(-1)^{|I|} \cdot Z\left(S_{\mathrm{C}_{I}}\right)=\left(\begin{array}{c}
\text { sum of all ways of gluing } \\
\text { all legs of } \mu(\mathrm{C}) \text { with } \ell_{S}(\mathrm{C}) / 2
\end{array}\right)+(\mathrm{i}-\mathrm{deg}>r),
$$

it being understood that the above sum is zero when $r$ is odd. 
This theorem generalizes the fact that the LMO invariant of rational homology 3spheres is universal among $\mathbb{Q}$-valued finite-type invariants in the sense of Ohtsuki and Goussarov and Habiro; see Le [13] and Habiro [10]. Indeed, finite-type invariants in this sense can be formulated in terms of $\mathbb{Z}$-LP surgeries; see Auclair and Lescop [1]. (A $\mathbb{Z}-$ LP surgery is defined in a way similar to a $\mathbb{Q}-$ LP surgery except that rational homology is replaced by integral homology.) However, the notion of "finite-type invariant" differs if one formulates it in terms of $\mathbb{Q}$-LP surgeries instead of $\mathbb{Z}$-LP surgeries: this difference has been recently analyzed by Moussard in the case of rational homology 3-spheres [23]. Let us observe that, in contrast with $\mathbb{Z}$-LP surgery, $\mathbb{Q}$-LP surgery relates any two rational homology 3-spheres: thus $\mathbb{Q}$-LP surgery is more appropriate if one wants to consider rational homology 3-spheres all together.

The analogue of the above theorem for the Kontsevich-Kuperberg-Thurston invariant $Z^{\mathrm{KKT}}$ has already been proved by Lescop: see [17; 19, Section 3]. However, it is not known whether we have the equality $Z^{\mathrm{KKT}}=Z$ in general. Lescop's "splitting formula" for $Z^{\mathrm{KKT}}$ generalizes her "sum formula" for the Casson-Walker invariant [18]. Indeed, according to $[16 ; 17]$, we have

$$
\text { i-degree } 2 \text { part of } Z^{\mathrm{KKT}}(S)=\frac{\lambda_{\mathrm{W}}(S)}{4} \cdot \cdots
$$

where $\lambda_{\mathrm{W}}(S)$ denotes Walker's extension of the Casson invariant as normalized in [26]. We shall prove the above theorem using the LMO functor $\tilde{Z}$ : this is a functorial extension of the LMO invariant to 3-manifolds with boundary, which was introduced in a previous joint work with Cheptea and Habiro [6]. The possibility of such a proof was announced in [6, Remark 7.12]. The main features of the LMO functor are recalled in Section 2 but, in a few words, let us recall that it is defined on the category of so-called "Lagrangian cobordisms" which are homology handlebodies with appropriate parameterizations of their boundaries, and it takes values in a certain category of Jacobi diagrams. We state in Section 3 a generalized version of the above theorem, where the $\mathbb{Q}$-homology 3-sphere $S$ is replaced by any $\mathbb{Q}$-Lagrangian cobordism $M$ and the LMO invariant $Z$ is replaced by the LMO functor $\widetilde{Z}$. This results in "generalized splitting formulas" involving a notion $\operatorname{Lk}_{M} \boldsymbol{E}(-,-)$ of a "linking number" in $M$, which depends on the choice of an isotropic subspace $\boldsymbol{E} \subset H_{1}(\partial M ; \mathbb{Q})$ such that $H_{1}(\partial M ; \mathbb{Q})=\boldsymbol{L}_{\boldsymbol{M}}^{\mathbb{Q}} \oplus \boldsymbol{E}$. Note that the category of Lagrangian cobordisms includes the monoid of homology cylinders, so that our results apply in particular to the LMO homomorphism (studied by Habiro and the author in [11] and the author and Meilhan in [20]) with a natural notion of linking number. The generalized splitting formulas are proved in Section 4 using the properties of the LMO functor established in [6]. The proof also needs several intermediate results, which can be of independent interest and are included in two appendices. Appendix A gives some properties of 
the generalized linking number $\operatorname{Lk}_{M}^{\boldsymbol{E}}(-,-)$ and it inspects the dependence on $\boldsymbol{E}$. Appendix B shows that the Milnor's triple linking numbers of an algebraically-split link in a $\mathbb{Q}$-homology 3-sphere are encoded in the "Y" part of the Kontsevich-LMO invariant. The latter result is in the continuity of the work of Habegger and Masbaum [9]; see also Moffatt [22].

Acknowledgements This work was partially supported by the French ANR research project ANR-08-JCJC-0114-01. We would like to thank Christine Lescop for her comments and for pointing out a small gap in a previous version of Appendix B.

Conventions The boundary $\partial N$ of an oriented manifold $N$ is always oriented using the "outward normal first" rule.

The boundary of a compact oriented 3-manifold $M$ is said to be parameterized by a closed oriented surface $F$ if $M$ comes with a continuous map $m: F \rightarrow M$ that is an orientation-preserving homeomorphism onto $\partial M$; the lower-case letter $m$ will also denote the corresponding homeomorphism $F \rightarrow \partial M$; we sometimes omit the boundary parameterization in our notation and denote the pair $(M, m)$ simply by $M$.

Implicitly, compact oriented 3-manifolds with parameterized boundary are considered up to homeomorphisms that preserve orientations and boundary parameterizations. Similarly, tangles in 3-manifolds are considered up to isotopy.

\section{Review of the LMO functor}

In this section, we briefly sketch the construction of the LMO functor. The reader is referred for further details to the paper [6]. Here our exposition is only intended to sum up the various steps of the construction using the same notation as in [6].

\subsection{The category of $\mathbb{Q}$-Lagrangian cobordisms}

We start by describing the source of the LMO functor and, for this, we consider the category $\mathcal{C} o b$ of 3-dimensional cobordisms introduced by Crane and Yetter [8]; see also Kerler [12]. By definition, an object of $\mathcal{C} o b$ is an integer $g \geq 0$, which one thinks of as the genus of a compact connected oriented surface with one boundary component. We actually fix a model $F_{g}$ for such a surface and we identify $\partial F_{g}$ with the square $S:=\partial\left([-1,1]^{2}\right)$. For any integers $g_{+} \geq 0$ and $g_{-} \geq 0$, a morphism $g_{+} \rightarrow g_{-}$in the category $\mathcal{C} o b$ is a cobordism $(M, m)$ from the surface $F_{g_{+}}$to the surface $F_{g_{-}}$: more 
precisely, $M$ is a compact connected oriented 3-manifold together with a boundary parameterization

$$
m:-F_{g-} \cup_{S \times\{-1\}}(S \times[-1,1]) \cup_{S \times\{1\}} F_{g_{+}} \longrightarrow M .
$$

Thus the boundary parameterization $m$ restricts to two embeddings $m_{-}: F_{g_{-}} \rightarrow M$ and $m_{+}: F_{g_{+}} \rightarrow M$, whose images are called bottom surface and top surface respectively. The composition $\circ$ in $\mathcal{C} o b$ is given by "vertical" gluing of cobordisms, while the "horizontal" gluing of cobordisms defines a strict monoidal structure $\otimes$ on that category. (Note that, to define the latter operation, we assume that the model surfaces $F_{0}, F_{1}, F_{2}, \ldots$ come with an identification of $F_{h+h^{\prime}}$ with $F_{h} \sharp_{\partial} F_{h^{\prime}}$ for any $h, h^{\prime} \geq 0$, where the boundary connected sum $\sharp_{\partial}$ is performed along the segments $\{1\} \times[-1,1] \subset \partial F_{h}$ and $\left.\{-1\} \times[-1,1] \subset \partial F_{h^{\prime}}.\right)$

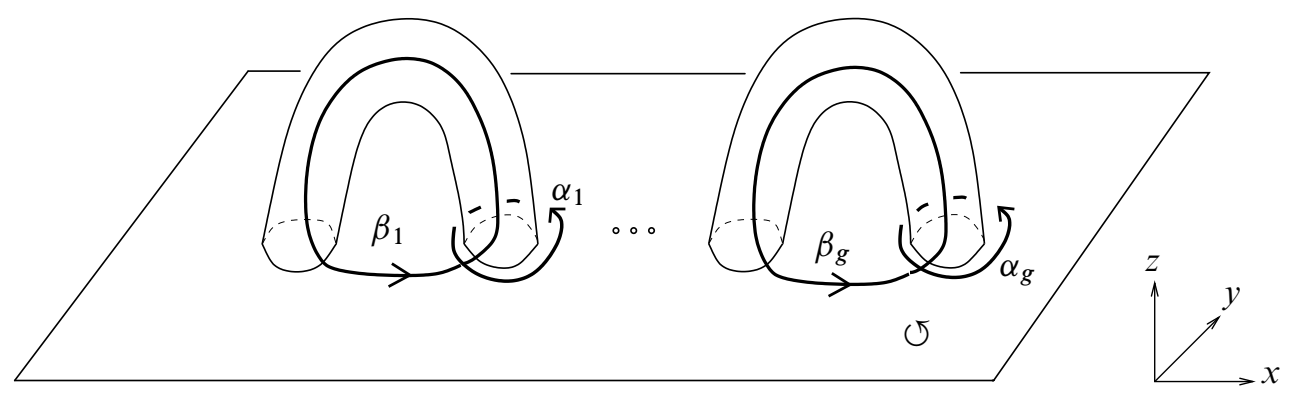

Figure 2.1: The model surface $F_{g}$ of genus $g \geq 0$, together with its system of meridians and parallels $(\alpha, \beta)$

The study of these cobordisms can be reduced to the study of some kind of tangles. To do this, we need to choose a system of "meridians" $\left(\alpha_{1}, \ldots, \alpha_{g}\right)$ and "parallels" $\left(\beta_{1}, \ldots, \beta_{g}\right)$ on each model surface $F_{g}$ (in a way compatible with the identifications $F_{h+h^{\prime}} \equiv F_{h} \sharp_{\partial} F_{h^{\prime}}$ for all $h, h^{\prime} \geq 0$ ). In order to fix ideas, we now assume that the surface $F_{g}$ is embedded in the ambient space $\mathbb{R}^{3} \subset S^{3}$ (with cartesian coordinates $x, y, z$ ): specifically, $F_{g}$ is obtained from the "horizontal" square $[-1,1] \times[-1,1] \times\{0\}$ by "adding" along the $x$-coordinate $g$ handles contained in the half-space $z>0$; see Figure 2.1 where the system of meridians and parallels $(\alpha, \beta)$ is also shown. Consider the submanifold $C_{g_{-}}^{g_{+}} \subset \mathbb{R}^{3}$ obtained from the standard cube $[-1,1]^{3}$ by "digging $g_{-}$ tunnels at the bottom side" and "attaching $g_{+}$handles on the top side", as shown in Figure 2.2. The oriented boundary of $C_{g_{-}}^{g_{+}}$consists of one copy of $F_{g_{+}}$and one copy of $-F_{g_{-}}$(which are obtained from the model surfaces by translation in the $z$ direction) joined by the cylinder $S \times[-1,1]$. Thus, for any $(M, m) \in \mathcal{C} o b\left(g_{+}, g_{-}\right)$, the source of the boundary parameterization $m$ is $\partial C_{g_{-}}^{g_{+}}$. The boundary of $M$ can be "killed" by attaching $\left(g_{-}+g_{+}\right)$handles of index 2 along $\partial M$ : more precisely, we attach one 
2-handle along each curve $m_{-}\left(\alpha_{i}\right)$ of the bottom surface and one 2-handle along each curve $m_{+}\left(\beta_{j}\right)$ of the top surface. What we get is a compact oriented 3-manifold $B$ with boundary parameterization $b: \partial C_{0}^{0} \rightarrow B$, ie a morphism $(B, b) \in \mathcal{C} o b(0,0)$. Furthermore, the cocores of the attached 2-handles define a $\left(g_{+}+g_{-}\right)$-component framed oriented tangle $\gamma$ in $B$ with components $\gamma_{1}^{+}, \ldots, \gamma_{g_{+}}^{+}$"on the top" and components $\gamma_{1}^{-}, \ldots, \gamma_{g_{-}}^{-}$"on the bottom": the pair $(B, \gamma)$ is called in [6] a bottom-top tangle. For example, if we do the previous operation on $C_{g_{-}}^{g_{+}} \in \mathcal{C} o b\left(g_{+}, g_{-}\right)$, then we obtain the trivial bottom-top tangle in the standard cube $C_{0}^{0}=[-1,1]^{3}$. There is a category ${ }_{b}^{t} \mathcal{T}$ of bottom-top tangles whose composition law is defined in such a way that the previous construction $(M, m) \mapsto((B, b), \gamma)$ defines an isomorphism of categories

$$
{ }_{b}^{t} \mathcal{T} \stackrel{\simeq}{\longrightarrow} \mathrm{Cob} \text {. }
$$

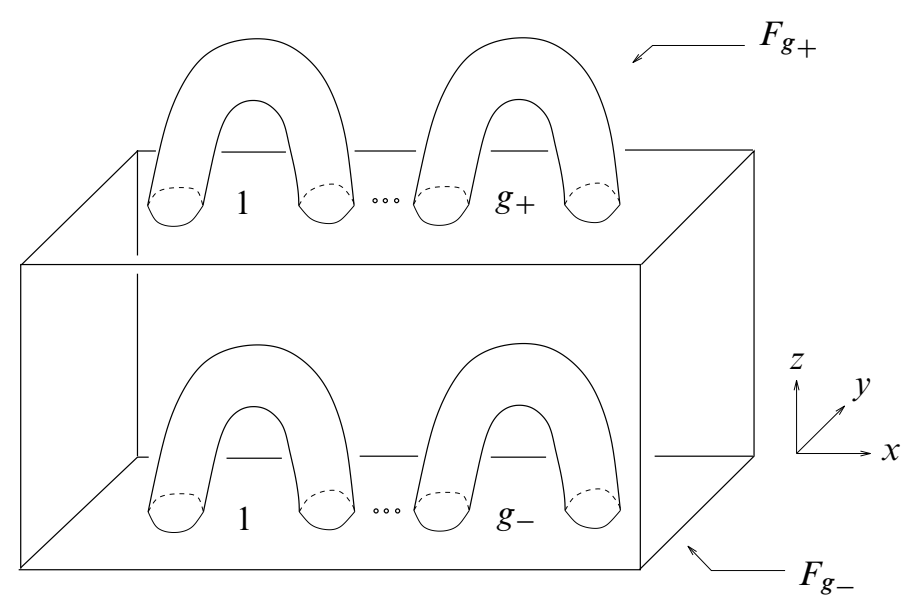

Figure 2.2: The cube $C_{g_{-}}^{g_{+}}$with $g_{-}$tunnels and $g_{+}$handles

Unfortunately, the LMO functor is not defined on the full category $\mathcal{C} o b$, but only on the subcategory $\mathbb{Q} \mathcal{L C}$ ob of $\mathbb{Q}$-Lagrangian cobordisms. The definition of this subcategory needs to $f i x$ a Lagrangian subspace $A_{g}^{\mathbb{Q}}$ of $H_{1}\left(F_{g} ; \mathbb{Q}\right)$ for any integer $g \geq 0$. A cobordism $(M, m) \in \mathcal{C} o b\left(g_{+}, g_{-}\right)$belongs to $\mathbb{Q} \mathcal{L C} o b\left(g_{+}, g_{-}\right)$if and only if:

(1) $H_{1}(M ; \mathbb{Q})=m_{-, *}\left(A_{g_{-}}^{\mathbb{Q}}\right)+m_{+, *}\left(H_{1}\left(F_{g_{+}} ; \mathbb{Q}\right)\right)$.

(2) $m_{+, *}\left(A_{g_{+}}^{\mathbb{Q}}\right) \subset m_{-, *}\left(A_{g_{-}}^{\mathbb{Q}}\right)$ as subspaces of $H_{1}(M ; \mathbb{Q})$.

Concretely, we take $A_{g}^{\mathbb{Q}}$ to be the subspace of $H_{1}\left(F_{g} ; \mathbb{Q}\right)$ spanned by $\left(\alpha_{1}, \ldots, \alpha_{g}\right)$, and we also consider the subspace $B_{g}^{\mathbb{Q}}$ spanned by $\left(\beta_{1}, \ldots, \beta_{g}\right)$. Then, in the presence of (2), condition (1) can be replaced by the following:

$\left(1^{\prime}\right) \quad m_{+, *} \oplus m_{-, *}: B_{g_{+}}^{\mathbb{Q}} \oplus A_{g_{-}}^{\mathbb{Q}} \rightarrow H_{1}(M ; \mathbb{Q})$ is an isomorphism. 
Example 2.1 Let $g \geq 0$ be an integer. A $\mathbb{Q}$-homology cylinder over the surface $F_{g}$ is a cobordism $(M, m) \in \mathcal{C} o b(g, g)$ such that $m_{ \pm, *}: H_{1}\left(F_{g} ; \mathbb{Q}\right) \rightarrow H_{1}(M ; \mathbb{Q})$ is an isomorphism and $m_{+, *}=m_{-, *}$. The set of $\mathbb{Q}$-homology cylinders constitutes a submonoid $\mathbb{Q} \mathcal{C} y l\left(F_{g}\right)$ of the monoid $\mathbb{Q} \mathcal{L C} o b(g, g)$.

Assume $((B, b), \gamma)$ is the tangle corresponding to a cobordism $(M, m) \in \mathcal{C} o b\left(g_{+}, g_{-}\right)$ via the description (2-1): then $(M, m)$ belongs to $\mathbb{Q} \mathcal{L C} o b\left(g_{+}, g_{-}\right)$if and only if $B$ is a $\mathbb{Q}$-homology cube (ie it has the same $\mathbb{Q}$-homology as the standard cube $[-1,1]^{3}=C_{0}^{0}$ ) and the linking matrix $\operatorname{Lk}_{B}\left(\gamma^{+}\right)$is trivial. Here the linking matrix $\operatorname{Lk}_{B}(\gamma)$ of the framed oriented tangle $\gamma$ in $B$ is defined by

$$
\begin{aligned}
\left(\begin{array}{cc}
\operatorname{Lk}_{B}\left(\gamma^{+}\right) & \operatorname{Lk}_{B}\left(\gamma^{+}, \gamma^{-}\right) \\
\operatorname{Lk}_{B}\left(\gamma^{-}, \gamma^{+}\right) & \operatorname{Lk}_{B}\left(\gamma^{-}\right)
\end{array}\right) & =\operatorname{Lk}_{B}(\gamma) \\
& :=\operatorname{Lk}_{\widehat{B}}(\widehat{\gamma})=\left(\begin{array}{cc}
\operatorname{Lk}_{\widehat{B}}\left(\hat{\gamma}^{+}\right) & \operatorname{Lk}_{\widehat{B}}\left(\hat{\gamma}^{+}, \hat{\gamma}^{-}\right) \\
\operatorname{Lk}_{\widehat{B}}\left(\hat{\gamma}^{-}, \hat{\gamma}^{+}\right) & \operatorname{Lk}_{\widehat{B}}\left(\hat{\gamma}^{-}\right)
\end{array}\right),
\end{aligned}
$$

where $\hat{\gamma}$ is the "plat" closure of $\gamma$ in the $\mathbb{Q}$-homology 3-sphere

$$
\widehat{B}:=B \cup_{b}\left(S^{3} \backslash \operatorname{int}\left(C_{0}^{0}\right)\right),
$$

and the rows/columns of $\operatorname{Lk}_{B}(\gamma)$ are indexed by the set $\pi_{0}(\gamma)=\pi_{0}\left(\gamma^{+}\right) \cup \pi_{0}\left(\gamma^{-}\right)$ of connected components of $\gamma$.

To be fully exact, the source of the LMO functor is the category $\mathbb{Q} \mathcal{L C} o b_{q}$ of $\mathbb{Q}$ Lagrangian $q$-cobordisms. An object of $\mathbb{Q} \mathcal{L C} o b_{q}$ is a nonassociative word in the single letter $\bullet$. For any two such words $w_{+}$and $w_{-}$, a morphism $w_{+} \rightarrow w_{-}$in the category $\mathbb{Q} \mathcal{L C} o b_{q}$ is a $\mathbb{Q}$-Lagrangian cobordism from $F_{g_{+}}$to $F_{g_{-}}$where $g_{ \pm}$is the length of $w_{ \pm}$. The category $\mathbb{Q} \mathcal{L} \mathcal{C} o b_{q}$ is a monoidal category in the nonstrict sense.

\subsection{The category of top-substantial Jacobi diagrams}

We now describe the target of the LMO functor and, for this, we need to fix some terminology. For any finite set $C$, we denote by $\mathcal{A}(C)$ the space of Jacobi diagrams colored by $C$. With the notation (1-3) of Section 1 , we have $\mathcal{A}(C):=\mathcal{A}(\mathbb{Q} \cdot C)$, where $\mathbb{Q} \cdot C$ is the vector space spanned by $C$. We shall also need the degree completion of $\mathcal{A}(C)$ which we denote in the same way. Here the degree of a Jacobi diagram is half the total number of its vertices; a Jacobi diagram of degree 1,

$$
\begin{gathered}
c_{2} \\
c_{1}
\end{gathered} \quad\left(\text { where } c_{1}, c_{2} \in C\right),
$$

is called a strut. Any rational matrix $M=\left(m_{i j}\right)_{i, j \in C}$, whose rows/columns are indexed by $C$, defines a linear combination of struts by setting

$$
M:=\sum_{i, j \in C} m_{i j} \vdots_{i}^{j} .
$$


If $S$ is another finite set, a Jacobi diagram in $\mathcal{A}(C \cup S)$ is $S$-substantial if it does not contain any strut whose two ends are colored by $S$.

The category of top-substantial Jacobi diagrams is the linear category ${ }^{t s} \mathcal{A}$ whose objects are integers $g \geq 0$ and whose space of morphisms ${ }^{t s} \mathcal{A}(g, f)$ is, for any integers $g \geq 0$ and $f \geq 0$, the subspace of $\mathcal{A}\left(\lfloor g\rceil^{+} \cup\lfloor f\rceil^{-}\right)$spanned by $\lfloor g\rceil^{+}$-substantial Jacobi diagrams. Here $\lfloor g\rceil^{+}$denotes the $g$-element finite set $\left\{1^{+}, \ldots, g^{+}\right\}$while $\lfloor f\rceil^{-}$ denotes the $f$-element finite set $\left\{1^{-}, \ldots, f^{-}\right\}$. For any integers $f, g, h \geq 0$, the composition law $\circ$ of ${ }^{t s} \mathcal{A}$ is defined for any Jacobi diagrams $D \in{ }^{t s} \mathcal{A}(g, f)$ and $E \in{ }^{t s} \mathcal{A}(h, g)$ by

$D \circ E:=\left(\begin{array}{c}\text { sum of all ways of gluing all the } i^{+} \text {-colored vertices of } D \\ \text { to all the } i^{-} \text {-colored vertices of } E \text {, for every } i=1, \ldots, g\end{array}\right) \in{ }^{t s} \mathcal{A}(h, f)$.

There is also a tensor product $\otimes$ in the category ${ }^{t} \mathcal{A}$ defined by the disjoint union of diagrams $\sqcup$ and the appropriate shifts of colors. Thus the category ${ }^{t s} \mathcal{A}$ is monoidal in the strict sense.

\subsection{Sketch of the construction}

The LMO functor of [6] is a tensor-preserving functor

$$
\tilde{Z}: \mathbb{Q} \mathcal{L} \operatorname{Cob}_{q} \longrightarrow{ }^{t s} \mathcal{A}
$$

Its construction uses the description (2-1) of cobordisms and it can be sketched as follows.

Let $(M, m) \in \mathbb{Q} \mathcal{L} \operatorname{Cob}_{q}(w, v)$ where $w$ and $v$ are nonassociative words in the single letter $\bullet$ of lengths $g$ and $f$ respectively. Let also $(B, \gamma):=((B, b), \gamma)$ be the bottom-top tangle in a $\mathbb{Q}$-homology cube corresponding to $M$ via (2-1). The framed tangle $\gamma$ can be promoted to a " $q$-tangle" in the sense of Le and Murakami $[14 ; 15]$ by transforming $w$ and $v$ into nonassociative words in the letters,+- by the rule $\bullet \mapsto(+-)$. Then the Kontsevich-LMO invariant of $(B, \gamma)$

$$
Z(B, \gamma) \in \mathcal{A}(\gamma)
$$

is defined in the (degree completion of the) space $\mathcal{A}(\gamma)$ of Jacobi diagrams based on the oriented 1-manifold underlying $\gamma$. The Kontsevich-LMO invariant $Z(B, \gamma)$ of $q$-tangles in $\mathbb{Q}$-homology cubes, which we are using here, has the following features:

- If $B$ is the standard cube $[-1,1]^{3}$, then $Z\left([-1,1]^{3}, \gamma\right)$ coincides with the usual Kontsevich integral $Z(\gamma)$ of $q$-tangles, as it is normalized in [6, Section 3.4]. (Note that this normalization differs from $[14 ; 15]$.) 
- If $\gamma$ is empty, then $Z(B, \varnothing)$ coincides with the usual LMO invariant $Z(\widehat{B})$ of the $\mathbb{Q}$-homology 3-sphere $\widehat{B}$. (Note that this invariant is denoted by $\widehat{\Omega}(\widehat{B})$ in [16] and is denoted by $\stackrel{\circ}{A}(\widehat{B})$ in $[2 ; 3]$.)

The Kontsevich-LMO invariant $Z(B, \gamma)$ is constructed from the usual Kontsevich integral $Z(L \cup \gamma) \in \mathcal{A}(L \cup \gamma)$, where $(L, \gamma)$ is a surgery presentation of $(B, \gamma)$ : thus, $L \subset[-1,1]^{3}$ is a framed link and $\gamma \subset[-1,1]^{3}$ is a framed oriented tangle disjoint from $L$ such that surgery along $L$ transforms $\left([-1,1]^{3}, \gamma\right)$ into $(B, \gamma)$. The passage $Z(L \cup \gamma) \rightsquigarrow Z(B, \gamma)$ can be performed in two ways: one can either follow the original LMO approach [16], or use the formal Gaussian integration of [2;3]. The latter approach is adopted in [6, Section 3.5]. Next, by applying the diagrammatic Poincaré-Birkhoff-Witt isomorphism $\chi: \mathcal{A}\left(\pi_{0}(\gamma)\right) \rightarrow \mathcal{A}(\gamma)$, we get

$$
\chi^{-1} Z(B, \gamma) \in \mathcal{A}\left(\pi_{0}(\gamma)\right)=\mathcal{A}\left(\lfloor g\rceil^{+} \cup\lfloor f\rceil^{-}\right),
$$

where the finite set $\pi_{0}(\gamma)=\pi_{0}\left(\gamma^{+}\right) \cup \pi_{0}\left(\gamma^{-}\right)$is identified with $\lfloor g\rceil^{+} \cup\lfloor f\rceil^{-}$in the obvious way. The fact that $\operatorname{Lk}_{B}\left(\gamma^{+}\right)=0$ implies that $\chi^{-1} Z(B, \gamma)$ is actually an element of ${ }^{t s} \mathcal{A}(g, f)$. Finally, the LMO functor is defined on the $\mathbb{Q}$-Lagrangian $q$-cobordism $M$ by

$$
\widetilde{Z}(M):=\chi^{-1} Z(B, \gamma) \circ \top_{g} \in{ }^{t s} \mathcal{A}(g, f),
$$

where $\mathrm{T}_{g} \in{ }^{t s} \mathcal{A}(g, g)$ is a constant that is defined in an appropriate way from the Baker-Campbell-Hausdorff series.

Finally, let us recall that the series of Jacobi diagrams $\widetilde{Z}(M) \in{ }^{t s} \mathcal{A}(g, f)$ can be decomposed as follows. Let $\mathcal{A}^{Y}\left(\lfloor g\rceil^{+} \cup\lfloor f\rceil^{-}\right)$be the subspace of $\mathcal{A}\left(\lfloor g\rceil^{+} \cup\lfloor f\rceil^{-}\right)$ spanned by Jacobi diagrams without strut component, and let $\mathcal{A}^{s}\left(\lfloor g\rceil^{+} \cup\lfloor f\rceil^{-}\right)$be the subspace spanned by disjoint unions of struts. Then we have

$$
\tilde{Z}(M)=\tilde{Z}^{s}(M) \sqcup \tilde{Z}^{Y}(M),
$$

where

$$
\tilde{Z}^{s}(M)=\exp _{\sqcup}\left(\frac{\operatorname{Lk}_{B}(\gamma)}{2}\right) \in \mathcal{A}^{s}\left(\lfloor g\rceil^{+} \cup\lfloor f\rceil^{-}\right), \quad \tilde{Z}^{Y}(M) \in \mathcal{A}^{Y}\left(\lfloor g\rceil^{+} \cup\lfloor f\rceil^{-}\right) .
$$

\section{Generalized splitting formulas for the LMO functor}

In this section, we state a generalized version of Lescop's splitting formulas. These "generalized splitting formulas" apply to the LMO functor of $\mathbb{Q}$-Lagrangian cobordisms. 


\subsection{Linking numbers in $\mathbb{Q}$-Lagrangian cobordisms}

The first lemma implies that, if one forgets about their boundary parameterizations, $\mathbb{Q}$-Lagrangian cobordisms are just $\mathbb{Q}$-homology handlebodies.

Lemma 3.1 Let $(M, m) \in \mathbb{Q} \mathcal{L C} o b(g, f)$ and let $N$ be a compact oriented 3-manifold which is embedded in $M$. If $N$ and $\partial N$ are connected, then $N$ is a $\mathbb{Q}$-homology handlebody.

Proof By doing the composition $C_{0}^{f} \circ M \circ C_{g}^{0}$ in the category $\mathbb{Q} \mathcal{L} \mathcal{C} o b$, we can assume $g=f=0$, ie $M$ is a $\mathbb{Q}$-homology cube. The map incl $: H_{1}(\partial N ; \mathbb{Q}) \rightarrow H_{1}(N ; \mathbb{Q})$ is surjective: for any $x=[X] \in H_{1}(N ; \mathbb{Q})$, there exists a rational 2-chain $Y$ in $M$ such that $\partial Y=X$ and $Y$ is transversal to $\partial N$; then, $X$ is homologous to $Y \cap \partial N$ in $N$. Therefore $H^{2}(N ; \mathbb{Q}) \simeq H_{1}(N, \partial N ; \mathbb{Q})=0$ which implies that $H_{2}(N ; \mathbb{Q})=0$. Let $r$ be the genus of the surface $\partial N$; since $\chi(N)=\chi(\partial N) / 2=1-r$, we deduce that $\operatorname{dim} H_{1}(N ; \mathbb{Q})=r$. Thus $N$ is a $\mathbb{Q}$-homology handlebody of genus $r$.

Therefore we can apply the results of Appendix A to any cobordism $M \in \mathbb{Q} \mathcal{L C} o b(g, f)$. Thus there is a notion of linking number $\operatorname{Lk}_{M}^{m_{*}(\boldsymbol{E})}(-,-)$ in $M$ for every choice of an $\boldsymbol{M}$-essential subspace $\boldsymbol{E}$ of

$$
H_{1}\left(\partial C_{f}^{g} ; \mathbb{Q}\right) \simeq \underbrace{H_{1}\left(F_{g} ; \mathbb{Q}\right)}_{\text {"top" }} \oplus \underbrace{H_{1}\left(F_{f} ; \mathbb{Q}\right)}_{\text {"bottom" }} .
$$

In particular, we are interested in the $M$-essential subspace

$$
\boldsymbol{B} \boldsymbol{A}:=\boldsymbol{B} \boldsymbol{A}(g, f)=B_{g}^{\mathbb{Q}} \oplus A_{f}^{\mathbb{Q}} \subset H_{1}\left(\partial C_{f}^{g} ; \mathbb{Q}\right)
$$

which is also isotropic. The resulting link invariant $\operatorname{Lk}_{M}(-,-):=\mathrm{Lk}_{M}^{m_{*}(\boldsymbol{B} \boldsymbol{A})}(-,-)$ can be reduced as follows to the usual notion of linking number in a $\mathbb{Q}$-homology 3-sphere.

Lemma 3.2 Let $M \in \mathbb{Q} \mathcal{L C} o b(g, f)$ and let $(B, \gamma) \in{ }_{b}^{t} \mathcal{T}(g, f)$ be the corresponding bottom-top tangle. Then, for any disjoint oriented knots $K, L \subset M \subset B$, we have

$$
\operatorname{Lk}_{M}(K, L)=\operatorname{Lk}_{\widehat{B}}(K, L),
$$

where $\widehat{B}$ is the $\mathbb{Q}$-homology 3-sphere defined by (2-2).

Proof Let $D$ be a rational 2-chain in $M$ such that $\partial D=L-\widetilde{L}$, where $\widetilde{L}$ is a rational $1-$ cycle in $\partial M$ such that $[\tilde{L}] \in H_{1}(\partial M ; \mathbb{Q})$ belongs to $m_{*}(\boldsymbol{B} A)$. Since each of the curves $m_{+}\left(\beta_{1}\right), \ldots, m_{+}\left(\beta_{g}\right)$ and $m_{-}\left(\alpha_{1}\right), \ldots, m_{-}\left(\alpha_{f}\right)$ bounds a disk in $B \subset \widehat{B}$, 
the 2-chain $D$ in $M$ can be "filled" to a 2-chain $D^{\prime}$ in $\widehat{B}$ such that $\partial D^{\prime}=L$ and $K \bullet_{\widehat{B}} D^{\prime}=K \bullet_{M} D$. Therefore we obtain

$$
\operatorname{Lk}_{M}(K, L) \stackrel{(\mathrm{A}-2)}{=} K \bullet_{M} D=K \cdot \widehat{B} D^{\prime}=\operatorname{Lk}_{\widehat{B}}(K, L) .
$$

Although the subspace $\boldsymbol{B} \boldsymbol{A}(g, f)$ has the advantage of being $M$-essential for any cobordism $M \in \mathbb{Q} \mathcal{L C} o b(g, f)$, it is sometimes more natural to use other essential subspaces.

Example 3.3 Consider the submonoid $\mathbb{Q} \mathcal{C} y l\left(F_{g}\right)$ of $\mathbb{Q} \mathcal{L C} o b(g, g)$ introduced in Example 2.1 and set $H_{\mathbb{Q}}:=H_{1}\left(F_{g} ; \mathbb{Q}\right)$. The following subspaces of $H_{1}\left(\partial C_{g}^{g} ; \mathbb{Q}\right) \simeq$ $H_{\mathbb{Q}} \oplus H_{\mathbb{Q}}$ are $M$-essential for any $M \in \mathbb{Q} \mathcal{C} y l\left(F_{g}\right)$ :

$$
\boldsymbol{D}:=\left\{(h, h) \mid h \in H_{\mathbb{Q}}\right\}, \quad \boldsymbol{E}_{+}:=\left\{(h, 0) \mid h \in H_{\mathbb{Q}}\right\}, \quad \boldsymbol{E}_{-}:=\left\{(0, h) \mid h \in H_{\mathbb{Q}}\right\} .
$$

The corresponding notions of linking number $\operatorname{Lk}_{M}^{m_{*}(\boldsymbol{D})}(-,-)$ and $\mathrm{Lk}_{M}^{m_{*}\left(\boldsymbol{E}_{ \pm}\right)}(-,-)$ coincide with the invariants denoted in $\left[20\right.$, Appendix B] by $\operatorname{Lk}(-,-)$ and $\operatorname{Lk}_{\mp}(-,-)$, respectively. We are particularly interested in the subspace $\boldsymbol{D}$ which is isotropic (in contrast with $\left.\boldsymbol{E}_{ \pm}\right)$. When $M=F_{g} \times[-1,1]$, the number $\operatorname{Lk}(K, L):=\operatorname{Lk}_{M}^{m_{*}(\boldsymbol{D})}(K, L)$ can be computed locally by considering knot diagrams on the bottom surface $F_{g} \times\{-1\}$ :

$$
\operatorname{Lk}(K, L):=\frac{1}{2}\left(\sharp^{K} \nwarrow \nearrow^{L}+\sharp^{L} \nwarrow \nearrow^{K}\right)-\frac{1}{2}\left(\sharp^{K} \nearrow^{L}+\sharp^{L} \nearrow^{K}\right)
$$

\subsection{Statement of the generalized splitting formulas}

Let $f, g \geq 0$ be integers. It follows from Lemma 3.2 and the equivalence (i) $\Leftrightarrow$ (iii) in Lemma A.6 that the $\mathbb{Q}$-LP surgery equivalence class of a cobordism $(M, m) \in$ $\mathbb{Q} \mathcal{L C} \operatorname{Cob}(g, f)$ is classified by the matrix $\operatorname{Lk}_{B}(\gamma)$, where $(B, \gamma) \in{ }_{b}^{t} \mathcal{T}(g, f)$ is the corresponding bottom-top tangle. According to the decomposition (2-3), this equivalence class is encoded by the "strut" part $\tilde{Z}^{s}(M)$ of $\tilde{Z}(M)$.

Thus we fix in the sequel a $\mathbb{Q}$-LP surgery equivalence class $\mathcal{M} \subset \mathbb{Q} \mathcal{L C}$ ob $(g, f)$ and consider only the "Y" part $\widetilde{Z}^{Y}$ of the LMO functor. Besides, we shall consider the following variants of $\widetilde{Z}^{Y}: \mathcal{M} \rightarrow \mathcal{A}^{Y}\left(\lfloor g\rceil^{+} \cup\lfloor f\rceil^{-}\right)$. Set $\boldsymbol{B} \boldsymbol{A}:=\boldsymbol{B} \boldsymbol{A}(g, f)$ and identify the vector spaces $\mathcal{A}^{Y}(\boldsymbol{B} \boldsymbol{A})$ and $\mathcal{A}^{Y}\left(\lfloor g\rceil^{+} \cup\lfloor f\rceil^{-}\right)$by the following correspondence of colors:

$$
\begin{aligned}
\boldsymbol{B} \boldsymbol{A}=B_{g}^{\mathbb{Q}} \oplus A_{f}^{\mathbb{Q}} \stackrel{\simeq}{\longrightarrow} \mathbb{Q} \cdot\left(\lfloor g\rceil^{+} \cup\lfloor f\rceil^{-}\right), \\
\beta_{j} \longmapsto j^{+}, \\
\alpha_{i} \longmapsto i^{-} .
\end{aligned}
$$


For any isotropic $\mathcal{M}$-essential subspace $\boldsymbol{E}$ of $H_{1}\left(\partial C_{f}^{g} ; \mathbb{Q}\right)$, consider the isomorphism $\kappa_{\mathcal{M}}^{\boldsymbol{B} \boldsymbol{A}, \boldsymbol{E}}: \mathcal{A}^{Y}(\boldsymbol{B} \boldsymbol{A}) \rightarrow \mathcal{A}^{Y}(\boldsymbol{E})$ introduced in Section A.4 and set

$$
Z_{\mathcal{M}}^{\boldsymbol{E}}:=\kappa_{\mathcal{M}}^{\boldsymbol{B A}, \boldsymbol{E}} \circ \tilde{Z}^{Y}: \mathcal{M} \longrightarrow \mathcal{A}^{Y}(\boldsymbol{E}) .
$$

Example 3.4 Take $\mathcal{M}:=\mathbb{Q} \mathcal{C} y l\left(F_{g}\right)$ which, by Lemma A.6, is the $\mathbb{Q}-$ LP surgery equivalence class of the standard cylinder $F_{g} \times[-1,1]$. Identify the space $\mathcal{A}^{Y}(\boldsymbol{D})$ with $\mathcal{A}^{Y}\left(H_{\mathbb{Q}}\right)$ where $\boldsymbol{D}$ is the $\mathcal{M}$-essential isotropic subspace discussed in Example 3.3 and is identified with $H_{\mathbb{Q}}=H_{1}\left(F_{g} ; \mathbb{Q}\right)$ by the map $h \mapsto(h / 2, h / 2)$. Let also $s: \mathcal{A}^{Y}\left(H_{\mathbb{Q}}\right) \rightarrow \mathcal{A}^{Y}\left(H_{\mathbb{Q}}\right)$ be the automorphism defined by $s(D):=(-1)^{\chi(D)} \cdot D$ where $\chi(D)$ is the Euler characteristic of an $H_{\mathbb{Q}}$-colored Jacobi diagram $D$. It is easily checked that $s \circ \kappa_{\mathcal{M}}^{\boldsymbol{B} \boldsymbol{A}, \boldsymbol{D}}$ is the isomorphism denoted by $\kappa$ in [20, Section 4.1]. Hence $s \circ Z_{\mathcal{M}}^{\boldsymbol{D}}$ coincides with the LMO homomorphism

$$
Z:=\kappa \circ \widetilde{Z}^{Y}: \mathbb{Q} \mathcal{C} y l\left(F_{g}\right) \longrightarrow \mathcal{A}^{Y}\left(H_{\mathbb{Q}}\right),
$$

which has been studied in $[11 ; 20]$.

We can now state our generalized splitting formulas.

Theorem 3.5 Let $\mathcal{M} \subset \mathbb{Q} \mathcal{L} \operatorname{Cob}(g, f)$ be a $\mathbb{Q}-L P$ surgery equivalence class and let $\boldsymbol{E}$ be an $\mathcal{M}$-essential isotropic subspace of $H_{1}\left(\partial C_{f}^{g} ; \mathbb{Q}\right)$. For any $M \in \mathcal{M}$ and for any finite family $C=\left(C_{1}, \ldots, C_{r}\right)$ of $\mathbb{Q}-L P$ pairs such that $C_{i}^{\prime} \subset \operatorname{int}(M)$ and $C_{i}^{\prime} \cap C_{j}^{\prime}=\varnothing$ for all $i \neq j$,

$$
\begin{aligned}
\sum_{I \subset\{1, \ldots, r\}}(-1)^{|I|} \cdot Z_{\mathcal{M}}^{E}\left(M_{\mathrm{C}_{I}}\right) \\
=\rho_{\mathrm{C}}^{E}\left(\begin{array}{c}
\text { sum of all ways of gluing } \\
\text { some legs of } \mu(\mathrm{C}) \text { with } \ell_{M}^{\boldsymbol{E}}(\mathrm{C}) / 2
\end{array}\right)+(\mathrm{i}-\mathrm{deg}>r)
\end{aligned}
$$

Here we have implicitly fixed some nonassociative words $w$ and $v$ in the single letter $\bullet$ of lengths $g$ and $f$, respectively, so that any $N \in \mathbb{Q} \mathcal{L C} o b(g, f)$ is upgraded to $N \in \mathbb{Q} \mathcal{L} \operatorname{Cob}_{q}(w, v)$. We have also used the same notation as in Section 1. Thus, for any $I \subset\{1, \ldots, r\}, M_{\mathrm{C}_{I}}$ is the manifold obtained from $M$ by the $\mathbb{Q}$-LP surgeries $M \rightsquigarrow M_{\mathrm{C}_{i}}$ performed simultaneously for all $i \in I$; the sum of Jacobi diagrams $\mu(\mathrm{C}) \in$ $\mathcal{A}\left(H_{1}(C ; \mathbb{Q})\right)$ and the symmetric bilinear form $\ell_{M}^{\boldsymbol{E}}(\mathrm{C}): H_{1}(C ; \mathbb{Q}) \times H_{1}(C ; \mathbb{Q}) \rightarrow \mathbb{Q}$ are defined as in Section 1 except that, for the latter, we use the generalized linking number $\operatorname{Lk}_{M}^{m_{*}(\boldsymbol{E})}(-,-)$ in $M$ instead of the usual linking number in a $\mathbb{Q}$-homology 3 -sphere (see Lemma A.1). Finally, $\rho_{\mathrm{C}}^{\boldsymbol{E}}: \mathcal{A}\left(H_{1}(C ; \mathbb{Q})\right) \rightarrow \mathcal{A}(\boldsymbol{E})$ is the linear map 
that changes the colors as follows:

$$
H_{1}(C ; \mathbb{Q}) \stackrel{\text { incl }_{*}}{\simeq} \bigoplus_{i=1}^{r} H_{1}\left(C_{i}^{\prime} ; \mathbb{Q}\right) \stackrel{\text { incl }_{*}}{\longrightarrow} H_{1}(M ; \mathbb{Q}) \stackrel{m_{*}}{\simeq} \boldsymbol{E} .
$$

Theorem 3.5 implies the theorem stated in Section 1. Indeed, any $\mathbb{Q}$-homology 3sphere $S$ can be transformed into a cobordism $M \in \mathbb{Q} \mathcal{L C} o b(0,0)$ by removing an open 3-ball. We take $\mathcal{M}:=\mathbb{Q} \mathcal{L C} \operatorname{ob}(0,0)$, which is the $\mathbb{Q}$-LP surgery equivalence class of the standard sphere $S^{3}$, and $\boldsymbol{E}:=0$. Then the linear map $\rho_{\mathrm{C}}^{\boldsymbol{E}}: \mathcal{A}\left(H_{1}(C ; \mathbb{Q})\right) \rightarrow \mathcal{A}(\varnothing)$ kills any diagram having at least one univalent vertex, so that the "sum of all ways of gluing some legs" turns into a "sum of all ways of gluing all legs".

Example 3.6 Take $\mathcal{M}:=\mathbb{Q} \mathcal{C} y l\left(F_{g}\right)$ and $\boldsymbol{E}:=\boldsymbol{D}$ as in Example 3.4. In this case, Theorem 3.5 provides splitting formulas for the LMO homomorphism $Z: \mathbb{Q} \mathcal{C} y l\left(F_{g}\right) \rightarrow$ $\mathcal{A}^{Y}\left(H_{\mathbb{Q}}\right)$ which involve the notion of linking number discussed in Example 3.3. For $r=2$, these formulas are generalizations of [20, Proposition C.2].

A consequence of Theorem 3.5 is that $Z_{\mathcal{M}}^{\boldsymbol{E}}$ is universal among $\mathbb{Q}$-valued finite-type invariants (in the sense of Ohtsuki, and Goussarov and Habiro). This universality property is obtained in [6, Section 7] in the special case $\boldsymbol{E}=\boldsymbol{B} \boldsymbol{A}$ and for $\mathbb{Z}$-Lagrangian cobordisms. Theorem 3.5 is proved in the next section by enhancing the arguments of [6, Theorem 7.11].

\section{Proof of the generalized splitting formulas}

This section is aimed at proving Theorem 3.5.

\subsection{A special case}

The following is the specialization of Theorem 3.5 for $\boldsymbol{E}=\boldsymbol{B} \boldsymbol{A}$.

Lemma 4.1 Let $w$ and $v$ be nonassociative words in the single letter $\bullet$ of lengths $g$ and $f$ respectively. Let $M \in \mathbb{Q} \mathcal{L} \operatorname{Cob}_{q}(w, v)$ and let $\mathrm{C}=\left(\mathrm{C}_{1}, \ldots, \mathrm{C}_{r}\right)$ be a family of $\mathbb{Q}-L P$ pairs, where $C_{i}^{\prime} \subset \operatorname{int}(M)$ and $C_{i}^{\prime} \cap C_{j}^{\prime}=\varnothing$ for all $i \neq j$. Then we have

$$
\begin{aligned}
\sum_{I \subset\{1, \ldots, r\}}(-1)^{|I|} \cdot \tilde{Z}^{Y}\left(M_{\mathrm{C}_{I}}\right) & \\
= & \rho_{\mathrm{C}}\left(\begin{array}{c}
\text { sum of all ways of gluing } \\
\text { some legs of } \mu(\mathrm{C}) \text { with } \ell_{M}(\mathrm{C}) / 2
\end{array}\right)+(\mathrm{i}-\mathrm{deg}>r) .
\end{aligned}
$$


Here we have set $\ell_{\boldsymbol{M}}(\mathrm{C}):=\ell_{\boldsymbol{M}}^{\boldsymbol{B} \boldsymbol{A}}(\mathrm{C})$ and the map $\rho_{\mathrm{C}}:=\rho_{\mathrm{C}}^{\boldsymbol{B} \boldsymbol{A}}: \mathcal{A}\left(H_{1}(C ; \mathbb{Q})\right) \rightarrow$ $\mathcal{A}(\boldsymbol{B} \boldsymbol{A}) \simeq \mathcal{A}\left(\lfloor g\rceil^{+} \cup\lfloor f\rceil^{-}\right)$consists of changing the colors of univalent vertices as follows:

$$
H_{1}(C ; \mathbb{Q}) \stackrel{\text { incl } *_{\simeq}}{\simeq} \bigoplus_{i=1}^{r} H_{1}\left(C_{i}^{\prime} ; \mathbb{Q}\right) \stackrel{\text { incl } *^{\longrightarrow}}{\longrightarrow} H_{1}(M ; \mathbb{Q}) \stackrel{m_{+, *} \oplus m_{-, *}}{\simeq} B_{g}^{\mathbb{Q}} \oplus A_{f}^{\mathbb{Q}}
$$

Using the notation of Lemma 4.1, we now show that formula (4-1) implies (3-2) for any isotropic $M$-essential subspace $\boldsymbol{E}$ of $H_{1}\left(\partial C_{f}^{g} ; \mathbb{Q}\right)$. Let $D \in \mathcal{A}\left(H_{1}(C ; \mathbb{Q})\right)$ be a Jacobi diagram of the form

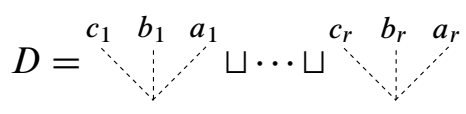

where $a_{1}, b_{1}, c_{1} \in H_{1}\left(C_{1} ; \mathbb{Q}\right), \ldots, a_{r}, b_{r}, c_{r} \in H_{1}\left(C_{r} ; \mathbb{Q}\right)$. Consider $k$ pairs of univalent vertices $\left\{v_{1}, w_{1}\right\}, \ldots,\left\{v_{k}, w_{k}\right\}$ of $D$ such that $\left\{v_{i}, w_{i}\right\} \cap\left\{v_{j}, w_{j}\right\}=\varnothing$ for any $i \neq j$. By making the identifications $v_{i} \equiv w_{i}$ for every $i \in\{1, \ldots, k\}$ and by applying the map (3-3) to the colors of the remaining univalent vertices, we obtain an $\boldsymbol{E}$-colored Jacobi diagram $D^{\prime}$. We can assume that $v_{i}$ and $w_{i}$ belong to different connected components of $D$ for all $i \in\{1, \ldots, k\}$ because $D^{\prime}$ is otherwise trivial in $\mathcal{A}(\boldsymbol{E})$ by the AS relation. For all $i \in\{1, \ldots, k\}$, let $V_{i}, W_{i} \subset C_{1}^{\prime} \sqcup \cdots \sqcup C_{r}^{\prime} \subset M$ be oriented knots representing

$$
\operatorname{color}\left(v_{i}\right), \operatorname{color}\left(w_{i}\right) \in H_{1}(C ; \mathbb{Q}) \simeq H_{1}\left(C_{1}^{\prime} ; \mathbb{Q}\right) \oplus \cdots \oplus H_{1}\left(C_{r}^{\prime} ; \mathbb{Q}\right),
$$

respectively, and let $f$ be the inverse of $m_{*}: \boldsymbol{B} \boldsymbol{A} \rightarrow H_{1}(M ; \mathbb{Q})$. The coefficient of $D^{\prime}$ in

$$
\rho_{\mathrm{C}}^{\boldsymbol{E}} \text { (sum of all ways of gluing some legs of } D \text { with } \ell_{\boldsymbol{M}}^{\boldsymbol{E}}(\mathrm{C}) / 2 \text { ) }
$$

is the product

$$
\begin{aligned}
\prod_{j=1}^{k} \operatorname{Lk}_{M}^{m_{*}(\boldsymbol{E})}\left(V_{j}, W_{j}\right) & \stackrel{(\mathrm{A}-4)}{=} \prod_{j=1}^{k}\left(\operatorname{Lk}_{M}\left(V_{j}, W_{j}\right)+\vartheta_{M}^{m_{*}(\boldsymbol{E})}\left(m_{*} f\left(\left[V_{j}\right]\right), m_{*} f\left(\left[W_{j}\right]\right)\right)\right) \\
& =\sum_{P \subset\{1, \ldots, k\}} \prod_{p \in P} \operatorname{Lk}_{M}\left(V_{p}, W_{p}\right) \cdot \prod_{q \notin P} \vartheta_{\mathcal{M}}^{\boldsymbol{E}}\left(f\left(\left[V_{q}\right]\right), f\left(\left[W_{q}\right]\right)\right) .
\end{aligned}
$$

This is also the coefficient of $D^{\prime}$ in

$$
\kappa_{\mathcal{M}}^{\boldsymbol{B} \boldsymbol{A}, \boldsymbol{E}} \rho_{\mathrm{C}}\left(\text { sum of all ways of gluing some legs of } D \text { with } \ell_{M}(\mathrm{C}) / 2\right)
$$


since the colors of $v_{i}$ and $w_{i}$ are mapped by (4-2) to $f\left(\left[V_{i}\right]\right)$ and $f\left(\left[W_{i}\right]\right)$ respectively. So (3-2) directly follows from (4-1) by applying $\kappa_{\mathcal{M}}^{\boldsymbol{B}}, \boldsymbol{E}$. The proof of formula (4-1) is postponed to Section 4.4. Before that, we need to establish a few technical results.

\subsection{From $\mathbb{Q}$-homology handlebodies to $\mathbb{Q}$-Lagrangian cobordisms}

The following will be useful to find appropriate boundary parameterizations of $\mathbb{Q}$ homology handlebodies.

Lemma 4.2 Let $\Sigma$ be a compact connected oriented surface of genus $g$ with $\partial \Sigma \cong S^{1}$, and let $L^{\mathbb{Q}}$ be a Lagrangian subspace of $H_{1}(\Sigma ; \mathbb{Q})$. Then there exists an orientationpreserving homeomorphism $s: F_{g} \rightarrow \Sigma$ such that $s_{*}\left(\alpha_{1}\right), \ldots, s_{*}\left(\alpha_{g}\right)$ span $L^{\mathbb{Q}}$.

Proof Let $L:=L^{\mathbb{Q}} \cap H_{1}(\Sigma ; \mathbb{Z})$. Then $L$ is an isotropic subgroup of $H_{1}(\Sigma ; \mathbb{Z})$, its rank is $\operatorname{dim}_{\mathbb{Q}}(L \otimes \mathbb{Q})=\operatorname{dim}_{\mathbb{Q}}\left(L^{\mathbb{Q}}\right)=g$ and the quotient $H_{1}(\Sigma ; \mathbb{Z}) / L$ is torsion-free. (Therefore $L$ is a Lagrangian subgroup of $H_{1}(\Sigma ; \mathbb{Z})$, ie it is isotropic maximal.)

Let $m=\left(m_{1}, \ldots, m_{g}\right)$ be a basis of the free abelian group $L$. Since $H_{1}(\Sigma ; \mathbb{Z}) / L$ is free, we can complement $m$ to a basis $(m, p)$ of $H_{1}(\Sigma ; \mathbb{Z})$. The matrix of the intersection pairing of $\Sigma$ in the basis $(m, p)$ is of the form

$$
\left(\begin{array}{cc}
0 & P^{t} \\
-P & Q
\end{array}\right)
$$

where $P$ is unimodular and $Q$ is antisymmetric. We write $Q$ as $R-R^{t}$, where $R$ is lower triangular, and we observe that

$$
\left(\begin{array}{cc}
I & 0 \\
P^{-1} R^{t}\left(P^{-1}\right)^{t} & P^{-1}
\end{array}\right) \cdot\left(\begin{array}{cc}
0 & P^{t} \\
-P & Q
\end{array}\right) \cdot\left(\begin{array}{cc}
I & P^{-1} R\left(P^{-1}\right)^{t} \\
0 & \left(P^{-1}\right)^{t}
\end{array}\right)=\left(\begin{array}{cc}
0 & I \\
-I & 0
\end{array}\right) .
$$

Thus we can complement $m$ to another basis $\left(m, p^{\prime}\right)$ of $H_{1}(\Sigma ; \mathbb{Z})$ with respect to which the matrix of the intersection pairing is

$$
\left(\begin{array}{cc}
0 & I \\
-I & 0
\end{array}\right)
$$

Then the isomorphism $H_{1}\left(F_{g} ; \mathbb{Z}\right) \rightarrow H_{1}(\Sigma ; \mathbb{Z})$ defined by $\alpha_{i} \mapsto m_{i}$ and $\beta_{i} \mapsto p_{i}^{\prime}$ preserves the intersection pairing. So, by a classical theorem of Dehn and Nielsen, we can realize this isomorphism by an orientation-preserving homeomorphism $s: F_{g} \rightarrow \Sigma$. The conclusion follows since, by construction, we have

$$
\left\langle s_{*}\left(\alpha_{1}\right), \ldots, s_{*}\left(\alpha_{g}\right)\right\rangle=\left\langle m_{1}, \ldots, m_{g}\right\rangle=L \subset H_{1}(\Sigma ; \mathbb{Z}) .
$$

We now explain how to turn $\mathbb{Q}$-homology handlebodies into $\mathbb{Q}$-Lagrangian cobordisms. 
Lemma 4.3 Let $C^{\prime}$ be a $\mathbb{Q}$-homology handlebody of genus $g$. Then there exists a boundary parameterization $c^{\prime}: \partial C_{0}^{g} \rightarrow C^{\prime}$ such that $\left(C^{\prime}, c^{\prime}\right) \in \mathbb{Q} \mathcal{L C} o b(g, 0)$.

Proof Let $\Sigma$ be the surface obtained from $\partial C^{\prime}$ by removing an open disk. The Lagrangian of $C^{\prime}$, namely

$$
\boldsymbol{L}_{C^{\prime}}^{\mathbb{Q}}=\operatorname{Ker}\left(\operatorname{incl}_{*}: H_{1}\left(\partial C^{\prime} ; \mathbb{Q}\right) \longrightarrow H_{1}\left(C^{\prime} ; \mathbb{Q}\right)\right),
$$

is a Lagrangian subspace of $H_{1}\left(\partial C^{\prime} ; \mathbb{Q}\right) \simeq H_{1}(\Sigma ; \mathbb{Q})$. So, by Lemma 4.2, we can find an orientation-preserving homeomorphism $s: F_{g} \rightarrow \Sigma$ such that $s_{*}\left(A_{g}^{\mathbb{Q}}\right)=L_{C^{\prime}}^{\mathbb{Q}}$. Next, viewing $\partial C_{0}^{g}$ as the union of $F_{g}$ with a closed disk, we can extend $s$ to an orientation-preserving homeomorphism $\partial C_{0}^{g} \rightarrow \partial C^{\prime}$ which, together with the inclusion $\partial C^{\prime} \subset C^{\prime}$, defines a boundary parameterization $c^{\prime}: \partial C_{0}^{g} \rightarrow C^{\prime}$. The cobordism $\left(C^{\prime}, c^{\prime}\right)$ is $\mathbb{Q}$-Lagrangian because:

(1) $H_{1}\left(C^{\prime} ; \mathbb{Q}\right)=0+\operatorname{incl}_{*}\left(H_{1}(\Sigma ; \mathbb{Q})\right)=c_{-, *}^{\prime}\left(A_{0}^{\mathbb{Q}}\right)+c_{+, *}^{\prime}\left(H_{1}\left(F_{g} ; \mathbb{Q}\right)\right)$.

(2) $c_{+, *}^{\prime}\left(A_{g}^{\mathbb{Q}}\right)=\operatorname{incl}_{*} \circ S_{*}\left(A_{g}^{\mathbb{Q}}\right)=\operatorname{incl}_{*}\left(L_{C^{\prime}}^{\mathbb{Q}}\right) \subset 0=c_{-, *}^{\prime}\left(A_{0}^{\mathbb{Q}}\right)$.

(For $(1)$, we have used the fact that $\operatorname{incl}_{*}: H_{1}\left(\partial C^{\prime} ; \mathbb{Q}\right) \rightarrow H_{1}\left(C^{\prime} ; \mathbb{Q}\right)$ is surjective.)

\subsection{The "Y" part of the LMO functor}

The next lemma relates triple cup products of closed oriented 3 -manifolds to $\widetilde{Z}_{1}$, the i-degree 1 part of the LMO functor.

Lemma 4.4 Let $C=\left(C^{\prime}, C^{\prime \prime}\right)$ be a $\mathbb{Q}-L P$ pair of genus $g$. Consider some boundary parameterizations

$$
c^{\prime}: \partial C_{0}^{g} \longrightarrow C^{\prime}, \quad c^{\prime \prime}: \partial C_{0}^{g} \longrightarrow C^{\prime \prime},
$$

that are compatible with the given identification $\partial C^{\prime} \equiv \partial C^{\prime \prime}$ and that satisfy $\left(C^{\prime}, c^{\prime}\right) \in$ $\mathbb{Q} \mathcal{L C} o b(g, 0)$ and $\left(C^{\prime \prime}, c^{\prime \prime}\right) \in \mathbb{Q} \mathcal{L C} o b(g, 0)$. Then the triple cup product form of the total manifold $C=\left(-C^{\prime}\right) \cup \cup_{\partial} C^{\prime \prime}$ is given by

$$
\mu(C)=\widetilde{Z}_{1}\left(C^{\prime}, c^{\prime}\right)-\widetilde{Z}_{1}\left(C^{\prime \prime}, c^{\prime \prime}\right) \in \mathcal{A}_{1}^{Y}\left(\lfloor g\rceil^{+}\right) \simeq \Lambda^{3} H_{1}(C ; \mathbb{Q}) .
$$

Here the isomorphism between $\mathcal{A}_{1}^{Y}\left(\lfloor g\rceil^{+}\right)$and $\Lambda^{3} H_{1}(C ; \mathbb{Q})$ is defined by

$$
\stackrel{k^{+} j^{+} i^{+}}{\because} \longmapsto\left[c_{+}^{\prime}\left(\beta_{i}\right)\right] \wedge\left[c_{+}^{\prime}\left(\beta_{j}\right)\right] \wedge\left[c_{+}^{\prime}\left(\beta_{k}\right)\right]
$$

Besides, the $\mathbb{Q}$-Lagrangian cobordisms $\left(C^{\prime}, c^{\prime}\right)$ and $\left(C^{\prime \prime}, c^{\prime \prime}\right)$ are equipped with an arbitrary nonassociative word of length $g$ in the single letter $\bullet$. 
Proof of Lemma 4.4 The existence of the boundary parameterizations $c^{\prime}$ and $c^{\prime \prime}$ follows from Lemma 4.3. Each of the closed 3-manifolds

$$
\left(-C_{0}^{g}\right) \cup_{c^{\prime}} C^{\prime}, \quad\left(-C_{0}^{g}\right) \cup_{c^{\prime \prime}} C^{\prime \prime} \quad \text { and } \quad C=\left(-C^{\prime}\right) \cup_{\partial} C^{\prime \prime}
$$

is contained in the singular 3-manifold $\left(-C_{0}^{g}\right) \cup_{c^{\prime} \sqcup c^{\prime \prime}}\left(C^{\prime} \sqcup C^{\prime \prime}\right)$. By computing triple cup products in this topological space, we find that

$$
\mu\left(\left(-C_{0}^{g}\right) \cup_{c^{\prime \prime}} C^{\prime \prime}\right)-\mu\left(\left(-C_{0}^{g}\right) \cup_{c^{\prime}} C^{\prime}\right)=\mu(C) \in \Lambda^{3} H_{1}(C ; \mathbb{Q}),
$$

where the homology groups are identified through the following isomorphisms (which are all induced by inclusions):

$$
\begin{aligned}
& H_{1}\left(\left(-C_{0}^{g}\right) \cup_{c^{\prime}} C^{\prime} ; \mathbb{Q}\right) \longleftarrow \simeq H_{1}\left(C^{\prime} ; \mathbb{Q}\right) \stackrel{\simeq}{\longrightarrow} H_{1}(C ; \mathbb{Q}), \\
& H_{1}\left(\left(-C_{0}^{g}\right) \cup_{c^{\prime \prime}} C^{\prime \prime} ; \mathbb{Q}\right) \longleftarrow H_{1}\left(C^{\prime \prime} ; \mathbb{Q}\right) \stackrel{\simeq}{\longrightarrow} H_{1}(C ; \mathbb{Q}) .
\end{aligned}
$$

Thus it suffices to show that $\mu\left(\left(-C_{0}^{g}\right) \cup_{c^{\prime}} C^{\prime}\right)=-\widetilde{Z}_{1}\left(C^{\prime}, c^{\prime}\right)$, and similarly for $\left(C^{\prime \prime}, c^{\prime \prime}\right)$.

In order to prove this identity, we consider the bottom-top tangle $(B, \gamma)$ corresponding to the cobordism $\left(C^{\prime}, c^{\prime}\right) \in \mathcal{C} o b(g, 0)$ by the correspondence (2-1). In this case, there is no bottom component in $\gamma$ (ie $\left.\gamma=\gamma^{+}\right)$and, since $\left(C^{\prime}, c^{\prime}\right)$ is $\mathbb{Q}$-Lagrangian, we have

$$
\operatorname{Lk}_{B}(\gamma)=\operatorname{Lk}_{\widehat{B}}(\widehat{\gamma})=0
$$

where $\hat{\gamma} \subset \widehat{B}$ is the plat closure of $\gamma$ in the $\mathbb{Q}$-homology 3-sphere $\widehat{B}$. Recall that $\tilde{Z}\left(C^{\prime}, c^{\prime}\right)$ is defined in [6] as a certain renormalization of the Kontsevich-LMO invariant $\chi^{-1} Z(B, \gamma)$; however this renormalization does not affect the "Y" part. Therefore, $\widetilde{Z}_{1}\left(C^{\prime}, c^{\prime}\right)$ is the i-degree 1 part of $\chi^{-1} Z(B, \gamma)$. Since the nondiagonal coefficients of $\operatorname{Lk}_{B}(\gamma)$ are trivial, the oriented link $\hat{\gamma}$ is algebraically split. Hence we can apply Lemma B.2 to deduce that $-\widetilde{Z}_{1}\left(C^{\prime}, c^{\prime}\right)$ is the linear combination of $Y$-shaped diagrams encoding Milnor's triple linking numbers of $\hat{\gamma}$ in $\hat{B}$. The closed oriented 3-manifold $\left(-C_{0}^{g}\right) \cup_{c^{\prime}} C^{\prime}$ is obtained from $\hat{B}$ by surgery along the framed link $\hat{\gamma}$. Since the $i^{\text {th }}$ diagonal coefficient of $\operatorname{Lk}_{B}(\gamma)$ - ie the framing number of $\hat{\gamma}_{i}$ - is trivial, the parallel of the framed knot $\hat{\gamma}_{i}$ is also the longitude of $\hat{\gamma}_{i}$ in the $\mathbb{Q}$-homology 3-sphere $\hat{B}$ : therefore, the surgery along the framed link $\hat{\gamma}$ is a longitudinal surgery. Remembering now the exact connection (B-1) between Milnor's triple linking numbers and triple cup products, we conclude that $\mu\left(\left(-C_{0}^{g}\right) \cup_{c^{\prime}} C^{\prime}\right)=-\widetilde{Z}_{1}\left(C^{\prime}, c^{\prime}\right)$. The same conclusion applies to $\left(C^{\prime \prime}, c^{\prime \prime}\right)$ with the same arguments. 


\subsection{Proof of the special case}

We can now prove Lemma 4.1, which will finish the proof of Theorem 3.5. Let $w$ and $v$ be nonassociative words in the single letter $\bullet$ of lengths $g$ and $f$ respectively. Let $(M, m) \in \mathbb{Q} \mathcal{L C} \operatorname{cob}_{q}(w, v)$ and let $\mathrm{C}=\left(\mathrm{C}_{1}, \ldots, \mathrm{C}_{r}\right)$ be a family of $\mathbb{Q}$-LP pairs, where $C_{i}^{\prime} \subset \operatorname{int}(M)$ and $C_{i}^{\prime} \cap C_{j}^{\prime}=\varnothing$ for all $i \neq j$.

We denote by $e_{1}, \ldots, e_{r}$ the genus of the $\mathbb{Q}$-homology handlebodies $C_{1}^{\prime}, \ldots, C_{r}^{\prime}$ respectively. We apply Lemma 4.3 to each of $C_{1}^{\prime}, \ldots, C_{r}^{\prime}$ and find boundary parameterizations $c_{1}^{\prime}, \ldots, c_{r}^{\prime}$ such that

$$
\left(C_{1}^{\prime}, c_{1}^{\prime}\right) \in \mathbb{Q} \mathcal{L} \operatorname{Cob}\left(e_{1}, 0\right), \quad \cdots, \quad\left(C_{r}^{\prime}, c_{r}^{\prime}\right) \in \mathbb{Q} \mathcal{L} \operatorname{Cob}\left(e_{r}, 0\right) .
$$

For any $i \in\{1, \ldots, r\}$, let $c_{i}^{\prime \prime}: \partial C_{0}^{e_{i}} \rightarrow C_{i}^{\prime \prime}$ be the boundary parameterization corresponding to $c_{i}^{\prime}$ through the identification $\partial C_{i}^{\prime} \equiv \partial C_{i}^{\prime \prime}$. Then, by definition of a $\mathbb{Q}-\mathrm{LP}$ pair, we also have

$$
\left(C_{1}^{\prime \prime}, c_{1}^{\prime \prime}\right) \in \mathbb{Q} \mathcal{L} \mathcal{C o b}\left(e_{1}, 0\right), \quad \ldots, \quad\left(C_{r}^{\prime \prime}, c_{r}^{\prime \prime}\right) \in \mathbb{Q} \mathcal{L C} \operatorname{cob}\left(e_{r}, 0\right) .
$$

We consider next a collar neighborhood $m_{-}\left(F_{f}\right) \times[-1,0]$ in $M$ of the bottom surface $m_{-}\left(F_{f}\right) \equiv m_{-}\left(F_{f}\right) \times\{-1\}$, we pick $r$ pairwise-disjoint closed disks on $m_{-}\left(F_{f}\right) \times\{0\}$ and we connect them to the disks $c_{1,-}^{\prime}\left(F_{0}\right) \subset \partial C_{1}^{\prime}, \ldots, c_{r,-}^{\prime}\left(F_{0}\right) \subset \partial C_{r}^{\prime}$ by pairwisedisjoint solid tubes $T_{1}, \ldots, T_{r}$ in the exterior of $\left(m_{-}\left(F_{f}\right) \times[-1,0]\right) \cup C_{1}^{\prime} \cup \cdots \cup C_{r}^{\prime}$. Thus we obtain a decomposition of $(M, m)$ in the monoidal category $\operatorname{Cob}$,

$$
(M, m)=\left(\left(C_{1}^{\prime}, c_{1}^{\prime}\right) \otimes \cdots \otimes\left(C_{r}^{\prime}, c_{r}^{\prime}\right) \otimes \operatorname{Id}_{f}\right) \circ(N, n),
$$

where $\operatorname{Id}_{f}$ denotes the identity of $f$ in $\mathcal{C} o b$ and $(N, n) \in \mathcal{C} o b(g, e+f)$ with $e:=e_{1}+$ $\cdots+e_{r}$. (Here $N$ corresponds to the exterior in $M$ of the union of $\left(m_{-}\left(F_{f}\right) \times[-1,0]\right)$, $C_{1}^{\prime} \cup \cdots \cup C_{r}^{\prime}$ and $\left.T_{1} \cup \cdots \cup T_{r}.\right)$ In fact, we have $(N, n) \in \mathbb{Q} \mathcal{L C} o b(g, e+f)$ so that (4-4) is actually a decomposition in the subcategory $\mathbb{Q} \mathcal{L C}$ ob. To check this, we consider the bottom-top tangles $(B, \gamma)$ and $(D, v)$ corresponding to $M$ and $N$ respectively. Then

$$
D=C_{0}^{e+f} \circ N \circ C_{g}^{0}=\left(\left(C_{0}^{e_{1}} \otimes \cdots \otimes C_{0}^{e_{r}}\right) \otimes C_{0}^{f}\right) \circ N \circ C_{g}^{0}
$$

can be obtained by $r \mathbb{Q}$-LP surgeries from

$$
\left(\left(C_{1}^{\prime} \otimes \cdots \otimes C_{r}^{\prime}\right) \otimes C_{0}^{f}\right) \circ N \circ C_{g}^{0} \stackrel{(4-4)}{=} C_{0}^{f} \circ M \circ C_{g}^{0}=B,
$$

and these surgeries transform the top tangle $v^{+} \subset D$ into the top tangle $\gamma^{+} \subset B$. The cobordism $M$ being $\mathbb{Q}$-Lagrangian, $B$ is a $\mathbb{Q}$-homology cube and $\operatorname{Lk}_{B}\left(\gamma^{+}\right)=0$. Since any $\mathbb{Q}$-LP surgery preserves the $\mathbb{Q}$-homology type as well as linking numbers (see Lemma A.5), we deduce that $D$ is a $\mathbb{Q}$-homology cube and $\operatorname{Lk}_{D}\left(v^{+}\right)=0$. Hence the cobordism $N$ is $\mathbb{Q}$-Lagrangian. 
In order to apply the LMO functor, we choose for every $i \in\{1, \ldots, r\}$ a nonassociative word $u_{i}$ of length $e_{i}$ in the single letter $\bullet$, with which we equip the $\mathbb{Q}$-Lagrangian cobordisms $\left(C_{i}^{\prime}, c_{i}^{\prime}\right)$ and $\left(C_{i}^{\prime \prime}, c_{i}^{\prime \prime}\right)$. The $\mathbb{Q}$-Lagrangian cobordism $(N, n)$ is equipped with the nonassociative word $\left(\cdots\left(\left(u_{1} u_{2}\right) u_{3}\right) \cdots u_{r}\right) v$. Then (4-4) is refined to the following decomposition in the category $\mathbb{Q} \mathcal{L C} o b_{q}$,

$$
M=\left(\left(\cdots\left(\left(C_{1}^{\prime} \otimes C_{2}^{\prime}\right) \otimes C_{3}^{\prime}\right) \cdots \otimes C_{r}^{\prime}\right) \otimes \operatorname{Id}_{v}\right) \circ N .
$$

For every subset $I \subset\{1, \ldots, r\}$, we have the same formula for the cobordism $M_{C_{I}}$ except that $C_{i}^{\prime}$ is now replaced by $C_{i}^{\prime \prime}$ for all $i \in I$. Then, by applying the tensorpreserving functor $\widetilde{Z}$, we obtain that

$$
\sum_{I \subset\{1, \ldots, r\}}(-1)^{|I|} \cdot \tilde{Z}\left(M_{C_{I}}\right)=\sum_{I \subset\{1, \ldots, r\}}(-1)^{|I|} \cdot\left(\tilde{Z}\left(C_{1}^{?}\right) \otimes \cdots \otimes \widetilde{Z}\left(C_{r}^{?}\right) \otimes \operatorname{Id}_{f}\right) \circ \tilde{Z}(N),
$$

where each question mark should be replaced by a prime or a double prime (depending on the subset $I$ ). Using the bilinearity of $\circ$ and $\otimes$ in the category ${ }^{t s} \mathcal{A}$, we deduce that

$$
\sum_{I \subset\{1, \ldots, r\}}(-1)^{|I|} \cdot \tilde{Z}\left(M_{C_{I}}\right)=\left(\left(\tilde{Z}\left(C_{1}^{\prime}\right)-\tilde{Z}\left(C_{1}^{\prime \prime}\right)\right) \otimes \cdots \otimes\left(\tilde{Z}\left(C_{r}^{\prime}\right)-\tilde{Z}\left(C_{r}^{\prime \prime}\right)\right) \otimes \operatorname{Id}_{f}\right) \circ \tilde{Z}(N)
$$

and, using Lemma 4.4, we get

$$
\sum_{I \subset\{1, \ldots, r\}}(-1)^{|I|} \cdot \tilde{Z}\left(M_{\mathrm{C}_{I}}\right)=\left(\mu\left(C_{1}\right) \otimes \cdots \otimes \mu\left(C_{r}\right) \otimes \operatorname{Id}_{f}\right) \circ \tilde{Z}(N)+(\mathrm{i}-\operatorname{deg}>r),
$$

where each $\mu\left(C_{i}\right)$ is regarded as an element of $\mathcal{A}^{Y}\left(\left\lfloor e_{i}\right\rceil^{+}\right) \subset{ }^{t s} \mathcal{A}\left(e_{i}, 0\right)$ by (4-3). For the sequel, it will be convenient to decompose the set $\lfloor e\rceil^{+}=\left\{1^{+}, \ldots, e^{+}\right\}$into

$$
\begin{array}{r}
\underbrace{\left\{1^{+}, \ldots, e_{1}^{+}\right\}}_{E_{1}} \cup \underbrace{\left\{\left(e_{1}+1\right)^{+}, \ldots,\left(e_{1}+e_{2}\right)^{+}\right\}}_{E_{2}} \\
\cup \cdots \cup \underbrace{\left\{\left(\sum_{i=1}^{r-1} e_{i}+1\right)^{+}, \ldots,\left(\sum_{i=1}^{r-1} e_{i}+e_{r}\right)^{+}\right\}}_{E_{r}} .
\end{array}
$$

Thus, by definition of the tensor product in ${ }^{t s} \mathcal{A}$, we obtain that

$$
\begin{aligned}
& \sum_{I \subset\{1, \ldots, r\}}(-1)^{|I|} \cdot \tilde{Z}\left(M_{C_{I}}\right) \\
& \stackrel{r}{=}\left(\mu\left(C_{1}\right) \sqcup \cdots \sqcup \mu\left(C_{r}\right) \sqcup \exp _{\sqcup}\left(\sum_{i=e+1}^{e+f} i^{i^{+}}\right)\right) \circ \tilde{Z}(N),
\end{aligned}
$$

where the symbol $\stackrel{r}{=}$ means an identity modulo terms of i-degree greater than $r$ and each $\mu\left(C_{i}\right)$ is now regarded as an element of $\mathcal{A}^{Y}\left(E_{i}\right)$. 
To proceed, we use again the bottom-top tangles $(B, \gamma)$ and $(D, v)$ corresponding to the cobordisms $M$ and $N$, respectively. According to (2-3), the "strut" part of $\widetilde{Z}(N)$ is $\exp _{\sqcup}\left(\operatorname{Lk}_{D}(v) / 2\right)$ so that (4-5) simplifies to

$$
\begin{aligned}
\sum_{I \subset\{1, \ldots, r\}} & (-1)^{|I|} \cdot \tilde{Z}\left(M_{C_{I}}\right) \\
& \stackrel{r}{=}\left(\mu\left(C_{1}\right) \sqcup \cdots \sqcup \mu\left(C_{r}\right) \sqcup \exp _{\sqcup}\left(\sum_{i=e+1}^{e+f} i^{i^{+}}\right)\right) \circ \exp _{\sqcup}\left(\operatorname{Lk}_{D}(v) / 2\right) .
\end{aligned}
$$

We denote by $\left(\delta_{1}, \ldots, \delta_{r}\right)$ the first $e$ components of the $(e+f)$-component tangle $v^{-}$. As we observed above, $D$ can be obtained from $B$ by $\mathbb{Q}$-LP surgeries: the tangles $v^{+}$ and $v^{-} \backslash \delta$ correspond through these surgeries to $\gamma^{+}$and $\gamma^{-}$, respectively. Since a $\mathbb{Q}-$ LP surgery preserves linking numbers, the symmetric matrix $\operatorname{Lk}_{D}(v)$ - whose rows and columns are indexed by $\pi_{0}\left(v^{+}\right) \cup \pi_{0}(\delta) \cup \pi_{0}\left(v^{-} \backslash \delta\right)$ - can be decomposed as

$$
\operatorname{Lk}_{D}(v)=\left(\begin{array}{ccc}
0 & \operatorname{Lk}_{D}\left(v^{+}, \delta\right) & \operatorname{Lk}_{B}\left(\gamma^{+}, \gamma^{-}\right) \\
\operatorname{Lk}_{D}\left(\delta, v^{+}\right) & \operatorname{Lk}_{D}(\delta) & \operatorname{Lk}_{D}\left(\delta, v^{-} \backslash \delta\right) \\
\operatorname{Lk}_{B}\left(\gamma^{-}, \gamma^{+}\right) & \operatorname{Lk}_{D}\left(v^{-} \backslash \delta, \delta\right) & \operatorname{Lk}_{B}\left(\gamma^{-}\right)
\end{array}\right) .
$$

Observe that the corner blocks of that matrix constitute $\operatorname{Lk}_{B}(\gamma)$. Next there exist simple combinatorial rules to compute compositions in the category ${ }^{t} \mathcal{A}$ of the form

$$
\left(\exp _{\sqcup}(H / 2) \sqcup h^{Y}\right) \circ\left(\exp _{\sqcup}(J / 2) \sqcup j^{Y}\right),
$$

where $H$ and $J$ are rational matrices (interpreted as linear combinations of struts) and $h^{Y}$ and $j^{Y}$ have no strut component; see [6, Lemma 4.5]. Applying these formulas to the right-hand side of (4-6), we obtain

$$
\begin{aligned}
\sum_{I \subset\{1, \ldots, r\}}(-1)^{|I|} \cdot \tilde{Z}\left(M_{C_{I}}\right) \\
\stackrel{r}{=} \exp _{\sqcup}\left(\operatorname{Lk}_{B}(\gamma) / 2\right) \sqcup \tilde{\rho}_{C}\left(\begin{array}{c}
\text { sum of all ways of gluing } \\
\text { some legs of } \mu(\mathrm{C}) \text { with } \operatorname{Lk}_{D}(\delta) / 2
\end{array}\right),
\end{aligned}
$$

where $\mu(\mathrm{C})=\mu\left(C_{1}\right) \sqcup \cdots \sqcup \mu\left(C_{r}\right)$ is regarded as an element of $\mathcal{A}^{Y}\left(\lfloor e\rceil^{+}\right)$, the symmetric matrix $\operatorname{Lk}_{D}(\delta) / 2$ is seen as a symmetric bilinear form on the vector space $\mathbb{Q} \cdot\lfloor e\rceil^{+}$and $\tilde{\rho}_{\mathrm{C}}: \mathcal{A}^{Y}\left(\lfloor e\rceil^{+}\right) \rightarrow \mathcal{A}^{Y}\left(\lfloor g\rceil^{+} \cup\lfloor f\rceil^{-}\right)$changes the colors as follows:

$$
l^{+} \longmapsto \sum_{j=1}^{g} \operatorname{Lk}_{D}\left(v_{j}^{+}, \delta_{l}\right) \cdot j^{+}+\sum_{i=1}^{f} \operatorname{Lk}_{D}\left(v_{e+i}^{-}, \delta_{l}\right) \cdot i^{-} \quad \text { for all } l^{+} \in\lfloor e\rceil^{+} \text {. }
$$

Since the strut part $\widetilde{Z}^{s}$ of $\tilde{Z}$ is preserved under $\mathbb{Q}$-LP surgery, we obtain that

$$
\sum_{I \subset\{1, \ldots, r\}}(-1)^{|I|} \cdot \tilde{Z}^{Y}\left(M_{\mathrm{C}_{I}}\right) \stackrel{r}{=} \tilde{\rho}_{\mathrm{C}}\left(\begin{array}{c}
\text { sum of all ways of gluing } \\
\text { some legs of } \mu(\mathrm{C}) \text { with } \operatorname{Lk}_{D}(\delta) / 2
\end{array}\right) .
$$


It remains to relate $\tilde{\rho}_{C}$ to $\rho_{C}$, and $\operatorname{Lk}_{D}(\delta)$ to $\ell_{M}(\mathrm{C})$. For the first relation, observe that we have the following identity in $H_{1}(M ; \mathbb{Q})$ for all $k \in\{1, \ldots, r\}$ and for all $l \in\left\{1, \ldots, e_{k}\right\}$, where we set $\bar{l}:=l+\sum_{s=1}^{k-1} e_{s} \in\{1, \ldots, e\}$ :

$$
\begin{aligned}
\operatorname{incl}_{*}\left(\left[c_{k,+}^{\prime}\left(\beta_{l}\right)\right]\right) & \\
= & \sum_{j=1}^{g} \operatorname{Lk}_{B}\left(c_{k,+}^{\prime}\left(\beta_{l}\right), \gamma_{j}^{+}\right) \cdot\left[m_{+}\left(\beta_{j}\right)\right]+\sum_{i=1}^{f} \operatorname{Lk}_{B}\left(c_{k,+}^{\prime}\left(\beta_{l}\right), \gamma_{i}^{-}\right) \cdot\left[m_{-}\left(\alpha_{i}\right)\right] \\
& =\sum_{j=1}^{g} \operatorname{Lk}_{D}\left(n_{-}\left(\beta_{\bar{l}}\right), v_{j}^{+}\right) \cdot\left[m_{+}\left(\beta_{j}\right)\right]+\sum_{i=1}^{f} \operatorname{Lk}_{D}\left(n_{-}\left(\beta_{\bar{l}}\right), v_{e+i}^{-}\right) \cdot\left[m_{-}\left(\alpha_{i}\right)\right] \\
& =\sum_{j=1}^{g} \operatorname{Lk}_{D}\left(\delta_{\bar{l}}, v_{j}^{+}\right) \cdot\left[m_{+}\left(\beta_{j}\right)\right]+\sum_{i=1}^{f} \operatorname{Lk}_{D}\left(\delta_{\bar{l}}, v_{e+i}^{-}\right) \cdot\left[m_{-}\left(\alpha_{i}\right)\right] .
\end{aligned}
$$

Thus, by comparing (4-7) to (4-2), we see that $\tilde{\rho}_{\mathrm{C}}$ corresponds to $\rho_{\mathrm{C}}$ through the isomorphism $\mathcal{A}^{Y}\left(\lfloor e\rceil^{+}\right) \simeq \mathcal{A}\left(H_{1}(C ; \mathbb{Q})\right)$ defined by the change of colors $\bar{l}^{+} \mapsto$ $\left[c_{k,+}^{\prime}\left(\beta_{l}\right)\right]$ for all $k \in\{1, \ldots, r\}$ and for all $l \in\left\{1, \ldots, e_{k}\right\}$. We now relate $\operatorname{Lk}_{D}(\delta)$ to $\ell_{M}(\mathrm{C})$ and, for this, we use the following notation: for any two colors $a, b \in\lfloor e\rceil^{+}$, we shall write $a \sim b$ if and only if $a, b$ belong to the same subset $E_{k}$ for some $k \in\{1, \ldots, r\}$. If $a \sim b$, then gluing an $a$-colored vertex to a $b$-colored vertex in $\mu(\mathrm{C})$ does not contribute to the right-hand side term of (4-8) due to the AS relation. Consider the case $a \nsim b$ : we assume that $a=\bar{u} \in E_{x}$ and $b=\bar{v} \in E_{y}$ with $x \neq y$, $u \in\left\lfloor e_{x}\right\rceil$ and $v \in\left\lfloor e_{y}\right\rceil$. Then we have

$$
\begin{aligned}
\left.\operatorname{Lk}_{D}\left(\delta_{a}, \delta_{b}\right)=\operatorname{Lk}_{D} \text { (parallel of } \delta_{a} \text {, parallel of } \delta_{b}\right) & =\operatorname{Lk}_{D}\left(n_{-}\left(\beta_{a}\right), n_{-}\left(\beta_{b}\right)\right) \\
& =\operatorname{Lk}_{B}\left(n_{-}\left(\beta_{a}\right), n_{-}\left(\beta_{b}\right)\right) \\
& =\operatorname{Lk}_{M}\left(c_{x,+}^{\prime}\left(\beta_{u}\right), c_{y,+}^{\prime}\left(\beta_{v}\right)\right),
\end{aligned}
$$

where the last equality follows from Lemma 3.2. Thus the nondiagonal blocks of the matrix of the pairing $\ell_{M}(\mathrm{C}): H_{1}(C ; \mathbb{Q}) \times H_{1}(C ; \mathbb{Q}) \rightarrow \mathbb{Q}$ in the basis

$$
\left(c_{1,+}^{\prime}\left(\beta_{1}\right), \ldots, c_{1,+}^{\prime}\left(\beta_{e_{1}}\right), \ldots, c_{r,+}^{\prime}\left(\beta_{1}\right), \ldots, c_{r,+}^{\prime}\left(\beta_{e_{r}}\right)\right)
$$

are the nondiagonal blocks of $\mathrm{Lk}_{D}(\delta)$. This concludes the proof of Lemma 4.1.

\section{Appendix A: Linking numbers in $\mathbb{Q}$-homology handlebodies}

In this appendix, we recall the definition of linking numbers in a $\mathbb{Q}$-homology handlebody, we study the ambiguity inherent in this definition and we prove a few properties which are needed to establish the splitting formulas in their full generality. 


\section{A.1 Definition}

We mainly follow Cimasoni and Turaev [7]. Let $M$ be a $\mathbb{Q}$-homology handlebody with Lagrangian $\boldsymbol{L}:=\boldsymbol{L}_{M}^{\mathbb{Q}} \subset H_{1}(\partial M ; \mathbb{Q})$. A subspace $\boldsymbol{E}$ of $H_{1}(\partial M ; \mathbb{Q})$ is said to be essential if the restriction of $\operatorname{incl}_{*}: H_{1}(\partial M ; \mathbb{Q}) \rightarrow H_{1}(M ; \mathbb{Q})$ to $\boldsymbol{E}$ is an isomorphism onto $H_{1}(M ; \mathbb{Q})$ or, equivalently, if $H_{1}(\partial M ; \mathbb{Q})=\boldsymbol{L} \oplus \boldsymbol{E}$; the corresponding section of incl $_{*}$ is denoted by the lower-case letter $e: H_{1}(M ; \mathbb{Q}) \rightarrow H_{1}(\partial M ; \mathbb{Q})$.

As explained in [7, Section 1], any essential subspace $\boldsymbol{E}$ of $H_{1}(\partial M ; \mathbb{Q})$ defines a notion of "generalized linking number" in $M$. Specifically, the linking number of two disjoint, oriented knots $K, L \subset \operatorname{int}(M)$ is the unique number $\operatorname{Lk}_{M}^{E}(K, L) \in \mathbb{Q}$ satisfying

$$
[L]=\mathrm{Lk}_{M}^{\boldsymbol{E}}(K, L) \cdot\left[m_{K}\right]+\operatorname{incl}_{*} e([L]) \in H_{1}(M \backslash K ; \mathbb{Q}) .
$$

Here $m_{K}$ is the oriented meridian of $K$, incl ${ }_{*}$ is induced by the inclusion $\partial M \subset M \backslash K$ and we use the following fact: the long exact sequence in homology for the pair $(M, M \backslash K)$ gives a short exact sequence

$$
0 \longrightarrow \mathbb{Q}\left[m_{K}\right] \longrightarrow H_{1}(M \backslash K ; \mathbb{Q}) \stackrel{\text { incl }_{*}}{\longrightarrow} H_{1}(M ; \mathbb{Q}) \longrightarrow 0 .
$$

The generalized linking number can be computed by the formula

$$
\operatorname{Lk}_{M}^{E}(K, L)=K \cdot{ }_{M} D,
$$

where $D$ is a rational 2-chain in $M$ transversal to $K$ such that the $1-$ cycle $\partial D-L$ is supported in $\partial M$ and represents an element of $\boldsymbol{E} \subset H_{1}(\partial M ; \mathbb{Q})$. It is easily observed (see [7, Section 1.3]) that

$$
\text { (A-3) } \quad \operatorname{Lk}_{M}^{\boldsymbol{E}}(L, K)-\mathrm{Lk}_{\boldsymbol{M}}^{\boldsymbol{E}}(K, L)=e([L]) \cdot{ }_{\partial M} e([K]),
$$

where $\cdot \partial M$ denotes the homology intersection in $\partial M$. In particular, the invariant $\operatorname{Lk}_{M}^{E}(-,-)$ is symmetric if $\boldsymbol{E}$ is isotropic (and hence Lagrangian) for the symplectic form $\cdot \partial M$.

\section{A.2 Dependence on the essential subspace}

Let $M$ be a $\mathbb{Q}$-homology handlebody with Lagrangian $\boldsymbol{L}:=\boldsymbol{L}_{M}^{\mathbb{Q}} \subset H_{1}(\partial M ; \mathbb{Q})$. We now study how generalized linking numbers depend on the choice of the essential subspace in $H_{1}(\partial M ; \mathbb{Q})$. First, we emphasize the homological nature of linking numbers. 
Lemma A.1 Let $P, Q \subset \operatorname{int}(M)$ be two submanifolds of dimension 3 such that $P \cap Q=\varnothing$, and let $\boldsymbol{E}$ be an essential subspace of $H_{1}(\partial M ; \mathbb{Q})$. Then there is a unique bilinear map $\ell_{\boldsymbol{M}}^{\boldsymbol{E}}: H_{1}(P ; \mathbb{Q}) \times H_{1}(Q ; \mathbb{Q}) \rightarrow \mathbb{Q}$ such that

$$
\ell_{M}^{\boldsymbol{E}}([K],[L])=\mathrm{Lk}_{M}^{\boldsymbol{E}}(K, L)
$$

for any oriented knots $K \subset P$ and $L \subset Q$.

Proof For $N=P$ or $Q$, denote by $\mathcal{K}_{N}$ the set of oriented knots in $N$ and observe that the map $\mathbb{Q} \times \mathcal{K}_{N} \rightarrow H_{1}(N ; \mathbb{Q})$ defined by $(p, K) \mapsto p[K]$ is surjective. Consider the map

$$
\tilde{\ell}_{M}^{\boldsymbol{E}}:\left(\mathbb{Q} \times \mathcal{K}_{P}\right) \times\left(\mathbb{Q} \times \mathcal{K}_{Q}\right) \longrightarrow \mathbb{Q}, \quad((p, K),(q, L)) \longmapsto p q \operatorname{Lk}_{M}^{\boldsymbol{E}}(K, L) .
$$

It follows from (A-1) that

$$
\tilde{\ell}_{\boldsymbol{M}}^{\boldsymbol{E}}((p, K),(q, L)) \cdot\left[m_{K}\right]=p \cdot(q[L])-p \cdot \operatorname{incl}_{*} e(q[L]) \in H_{1}(M \backslash K ; \mathbb{Q}),
$$

which shows that, for fixed $(p, K)$, the number $\tilde{\ell}_{M}^{\boldsymbol{E}}((p, K),(q, L))$ only depends (linearly) on $q[L] \in H_{1}(Q ; \mathbb{Q})$. Furthermore, we have

$$
\tilde{\ell}_{\boldsymbol{M}}^{\boldsymbol{E}}((p, K),(q, L)) \stackrel{(\mathrm{A}-3)}{=} \tilde{\ell}_{\boldsymbol{M}}^{\boldsymbol{E}}((q, L),(p, K))+e(q[K]) \cdot{ }_{\partial M} e(p[L]),
$$

so that, for a fixed $(q, L), \tilde{\ell}_{M}^{E}((p, K),(q, L))$ only depends (linearly) on the class $p[K] \in H_{1}(P ; \mathbb{Q})$. Thus $\tilde{\ell}_{\boldsymbol{M}}^{\boldsymbol{E}}$ factorizes to a unique bilinear map $\ell_{\boldsymbol{M}}^{\boldsymbol{E}}: H_{1}(P ; \mathbb{Q}) \times$ $H_{1}(Q ; \mathbb{Q}) \rightarrow \mathbb{Q}$.

Next, we define a bilinear pairing in $H_{1}(\partial M ; \mathbb{Q})$.

Lemma A.2 Let $\boldsymbol{E}$ be an essential subspace of $H_{1}(\partial M ; \mathbb{Q})$. The generalized linking number $\operatorname{Lk}_{\boldsymbol{M}}^{\boldsymbol{E}}(-,-)$ induces a bilinear form $\vartheta_{\boldsymbol{M}}^{\boldsymbol{E}}: H_{1}(\partial M ; \mathbb{Q}) \times H_{1}(\partial M ; \mathbb{Q}) \rightarrow \mathbb{Q}$. Moreover, we have that the left radical and right radical of $\vartheta_{M}^{\boldsymbol{E}}$ are given by $\left\{x \in H_{1}(\partial M ; \mathbb{Q}) \mid \vartheta_{M}^{\boldsymbol{E}}(x,-)=0\right\}=\boldsymbol{L}, \quad\left\{y \in H_{1}(\partial M ; \mathbb{Q}) \mid \vartheta_{\boldsymbol{M}}^{\boldsymbol{E}}(-, y)=0\right\}=\boldsymbol{E}$, respectively, and $\vartheta_{\boldsymbol{M}}^{\boldsymbol{E}}$ is the opposite of the intersection form $\bullet_{\partial M}$ on $\boldsymbol{E} \times \boldsymbol{L}$.

Proof Consider a collar neighborhood $\partial M \times[-1,0]$ of $\partial M \equiv \partial M \times\{0\}$. By applying Lemma A.1 to $P=\partial M \times]-1,-\frac{1}{2}[$ and $Q=\partial M \times]-\frac{1}{2}, 0$, we get a bilinear map $\vartheta_{M}^{\boldsymbol{E}}: H_{1}(\partial M ; \mathbb{Q}) \times H_{1}(\partial M ; \mathbb{Q}) \rightarrow \mathbb{Q}$.

Set ${ }^{*} R:=\left\{x \in H_{1}(\partial M ; \mathbb{Q}) \mid \vartheta_{M}^{\boldsymbol{E}}(x,-)=0\right\}, R^{*}:=\left\{y \in H_{1}(\partial M ; \mathbb{Q}) \mid \vartheta_{M}^{\boldsymbol{E}}(-, y)=0\right\}$. We show that $\boldsymbol{E} \subset R^{*}$. Let $y \in \boldsymbol{E}$ and let $\left.L \subset \partial M \times\right]-\frac{1}{2}, 0[$ be an oriented knot 
representing $y$ in $H_{1}(\partial M \times]-\frac{1}{2}, 0[; \mathbb{Q}) \simeq H_{1}(\partial M ; \mathbb{Q})$. Then, for any oriented knot $K \subset \partial M \times]-1,-\frac{1}{2}[$, we have

$$
\operatorname{Lk}_{M}^{\boldsymbol{E}}(K, L) \cdot\left[m_{K}\right] \stackrel{(\mathrm{A}-1)}{=}[L]-\operatorname{incl}_{*} e([L])=[L]-[L]=0 \in H_{1}(M \backslash K ; \mathbb{Q})
$$

so that $\operatorname{Lk}_{M}^{\boldsymbol{E}}(K, L)=0$; this implies that $\vartheta_{M}^{\boldsymbol{E}}(-, y)=0$.

We show that $\vartheta_{M}^{\boldsymbol{E}}(\boldsymbol{L}, \boldsymbol{L})=0$ which, by the previous paragraph, implies $\boldsymbol{L} \subset{ }^{*} R$. Let $x, y \in \boldsymbol{L} \subset H_{1}(\partial M ; \mathbb{Q}) \simeq H_{1}(\partial M \times[-1,0] ; \mathbb{Q})$ and represent them by some oriented knots $K \subset \partial M \times]-1,-\frac{1}{2}[, L \subset \partial M \times]-\frac{1}{2}, 0[$, respectively. It follows from (A-3) that $\vartheta_{M}^{\boldsymbol{E}}(x, y)=\operatorname{Lk}_{\boldsymbol{M}}^{\boldsymbol{E}}(K, L)=\operatorname{Lk}_{M}^{\boldsymbol{E}}(L, K)$. Since $\left.\left.[K]=0 \in H_{1}\left(M \backslash(\partial M \times]-\frac{1}{2}, 0\right]\right) ; \mathbb{Q}\right)$, we have $[K]=0 \in H_{1}(M \backslash L ; \mathbb{Q})$ so that $\operatorname{Lk}_{M}^{E}(L, K)=0$.

We prove that $\vartheta_{M}^{\boldsymbol{E}}$ coincides with the opposite of $\bullet_{\partial \boldsymbol{M}}$ on $\boldsymbol{E} \times \boldsymbol{L}$. Let $x, y \in$ $H_{1}(\partial M ; \mathbb{Q}) \simeq H_{1}(\partial M \times[-1,0] ; \mathbb{Q})$ such that $x \in \boldsymbol{E}$ and $y \in \boldsymbol{L}$. We represent $x, y$ by oriented knots $K \subset \partial M \times]-1,-\frac{1}{2}[$ and $L \subset \partial M \times]-\frac{1}{2}, 0$, respectively. Choose a rational 2-chain $D$ transversal to $K$ such that $\partial D=L$. Then

$$
\vartheta_{\boldsymbol{M}}^{\boldsymbol{E}}(x, y)=\mathrm{Lk}_{\boldsymbol{M}}^{\boldsymbol{E}}(K, L) \stackrel{(\mathrm{A}-2)}{=} K \bullet_{M} D=-x \cdot \partial M y .
$$

We now prove that ${ }^{*} R \subset \boldsymbol{L}$ and $R^{*} \subset \boldsymbol{E}$. Denote by $p_{\boldsymbol{E}}: H_{1}(\partial M ; \mathbb{Q}) \rightarrow \boldsymbol{L}$ and $q_{\boldsymbol{E}}: H_{1}(\partial M ; \mathbb{Q}) \rightarrow \boldsymbol{E}$ the projections of the direct sum $H_{1}(\partial M ; \mathbb{Q})=\boldsymbol{L} \oplus \boldsymbol{E}$. Let $x \in H_{1}(\partial M ; \mathbb{Q})$ such that $\vartheta_{M}^{\boldsymbol{E}}(x,-)=0$; then, for all $l \in \boldsymbol{L}$,

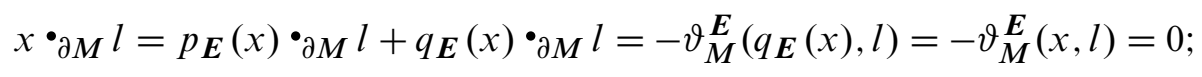

since $\boldsymbol{L}$ is Lagrangian with respect to ${ }^{\partial} \partial \boldsymbol{M}$, it follows that $x \in \boldsymbol{L}$. We conclude that ${ }^{*} R=\boldsymbol{L}$. Let $y \in H_{1}(\partial M ; \mathbb{Q})$ such that $\vartheta_{\boldsymbol{M}}^{\boldsymbol{E}}(-, y)=0$; then, for all $x \in H_{1}(\partial M ; \mathbb{Q})$,

$$
\begin{aligned}
x \bullet_{\partial M} p_{\boldsymbol{E}}(y) & =p_{\boldsymbol{E}}(x) \bullet_{\partial M} p_{\boldsymbol{E}}(y)+q_{\boldsymbol{E}}(x) \bullet_{\partial M} p_{\boldsymbol{E}}(y) \\
& =-\vartheta_{\boldsymbol{M}}^{\boldsymbol{E}}\left(q_{\boldsymbol{E}}(x), p_{\boldsymbol{E}}(y)\right)=-\vartheta_{\boldsymbol{M}}^{\boldsymbol{E}}\left(q_{\boldsymbol{E}}(x), y\right)=0 .
\end{aligned}
$$

We deduce that $p_{\boldsymbol{E}}(y)=0$, ie $y \in \boldsymbol{E}$. We conclude that $R^{*}=\boldsymbol{E}$.

The form $\vartheta_{\boldsymbol{M}}^{\boldsymbol{E}}$ is the "generalized Seifert form" of the surface $-\partial M$ as defined in [7, Section 2]. It is not symmetric, but it satisfies the following: for all $x, y \in H_{1}(\partial M ; \mathbb{Q})$,

$$
\vartheta_{M}^{\boldsymbol{E}}(y, x)-\vartheta_{M}^{\boldsymbol{E}}(x, y)=e\left(\operatorname{incl}_{*}(y)\right) \bullet_{\partial M} e\left(\operatorname{incl}_{*}(x)\right)-y \cdot \partial M x .
$$

(This can be deduced from identity (A-3); see also [7, Section 2.1].) This form measures how the generalized linking number depends on the choice of the essential subspace, as the next lemma shows. 
Lemma A.3 Let $\boldsymbol{E}, \boldsymbol{F}$ be essential subspaces of $H_{1}(\partial M ; \mathbb{Q})$. For any disjoint, oriented knots $K, L \subset \operatorname{int}(M)$, we have

$$
\text { (A-4) } \quad \operatorname{Lk}_{M}^{\boldsymbol{F}}(K, L)=\operatorname{Lk}_{M}^{\boldsymbol{E}}(K, L)-\vartheta_{\boldsymbol{M}}^{\boldsymbol{E}}(f([K]), f([L])),
$$

where $f: H_{1}(M ; \mathbb{Q}) \rightarrow H_{1}(\partial M ; \mathbb{Q})$ is the section of the map incl $_{*}: H_{1}(\partial M ; \mathbb{Q}) \rightarrow$ $H_{1}(M ; \mathbb{Q})$ corresponding to $\boldsymbol{F}$.

Proof Consider a collar neighborhood $\partial M \times[-1,0]$ of $\partial M \equiv \partial M \times\{0\}$, which does not cut $K \cup L$. Let $\widetilde{K} \subset \partial M \times]-1,-\frac{1}{2}[$ and $\widetilde{L} \subset \partial M \times]-\frac{1}{2}, 0[$ be oriented knots such that

$$
\begin{aligned}
& {[\widetilde{K}]=f([K]) \in H_{1}(\partial M \times]-1,-\frac{1}{2}[; \mathbb{Q}) \simeq H_{1}(\partial M ; \mathbb{Q}),} \\
& {[\widetilde{L}]=f([L]) \in H_{1}(\partial M \times]-\frac{1}{2}, 0[; \mathbb{Q}) \simeq H_{1}(\partial M ; \mathbb{Q}) .}
\end{aligned}
$$

We have to prove that

$$
\operatorname{Lk}_{M}^{\boldsymbol{E}}(\tilde{K}, \tilde{L})=\mathrm{Lk}_{M}^{\boldsymbol{E}}(K, L)-\mathrm{Lk}_{M}^{\boldsymbol{F}}(K, L) .
$$

Since $\tilde{K}$ is rationally homologous to $K$ in the exterior of $\left.\partial M \times]-\frac{1}{2}, 0\right]$ and since $\widetilde{L}$ is contained in $\left.\partial M \times]-\frac{1}{2}, 0\right], \widetilde{K}$ is rationally homologous to $K$ in $M \backslash \widetilde{L}$. Therefore

$$
\operatorname{Lk}_{M}^{E}(\tilde{K}, \tilde{L})=\operatorname{Lk}_{M}^{E}(K, \tilde{L})
$$

and we obtain

$$
\begin{aligned}
\operatorname{Lk}_{M}^{\boldsymbol{E}}(\tilde{K}, \tilde{L}) \cdot\left[m_{K}\right] & =[\tilde{L}]-\operatorname{incl}_{*} e([\tilde{L}]) \\
& =\operatorname{incl}_{*} f([L])-\operatorname{incl}_{*} e([L]) \in H_{1}(M \backslash K ; \mathbb{Q}) .
\end{aligned}
$$

Besides, we have

$$
\begin{aligned}
\operatorname{Lk}_{M}^{\boldsymbol{E}}(K, L) \cdot\left[m_{K}\right]-\mathrm{Lk}_{M}^{\boldsymbol{F}}(K, L) \cdot\left[m_{K}\right] & =\left([L]-\operatorname{incl}_{*} e([L])\right)-\left([L]-\operatorname{incl}_{*} f([L])\right) \\
& =\operatorname{incl}_{*} f([L])-\operatorname{incl}_{*} e([L]) \in H_{1}(M \backslash K ; \mathbb{Q}) .
\end{aligned}
$$

Identity (A-5) follows.

\section{A.3 Essential Jacobi diagrams}

Let $M$ be a $\mathbb{Q}$-homology handlebody. For any essential subspace $\boldsymbol{E}$ of $H_{1}(\partial M ; \mathbb{Q})$, we consider the subspace $\mathcal{A}^{Y}(\boldsymbol{E}) \subset \mathcal{A}(\boldsymbol{E})$ spanned by Jacobi diagrams without strut components. If $\boldsymbol{F} \subset H_{1}(\partial M ; \mathbb{Q})$ is another essential subspace, we would like to identify $\mathcal{A}^{Y}(\boldsymbol{E})$ and $\mathcal{A}^{Y}(\boldsymbol{F})$ in a canonical way. Of course, there is the obvious isomorphism $\rho_{M}^{\boldsymbol{F}, \boldsymbol{E}}: \mathcal{A}^{Y}(\boldsymbol{F}) \rightarrow \mathcal{A}^{Y}(\boldsymbol{E})$ that consists of changing the colors of univalent vertices by means of the isomorphism $e f^{-1}: \boldsymbol{F} \stackrel{\simeq}{\longrightarrow} H_{1}(M ; \mathbb{Q}) \stackrel{\simeq}{\longrightarrow} \boldsymbol{E}$. But this is not enough for our purposes. 
To go further, we assume that $\boldsymbol{E}$ and $\boldsymbol{F}$ are also isotropic subspaces of $H_{1}(\partial M ; \mathbb{Q})$. Then the restriction of the form $\vartheta_{M}^{\boldsymbol{E}}$ to $\boldsymbol{F} \times \boldsymbol{F}$ is symmetric by Lemma A.3. So we can consider the linear map

$$
\kappa_{M}^{\boldsymbol{F}, \boldsymbol{E}}: \mathcal{A}^{Y}(\boldsymbol{F}) \longrightarrow \mathcal{A}^{Y}(\boldsymbol{E})
$$

defined for any $\boldsymbol{F}$-colored Jacobi diagram $D$ by

$$
\kappa_{M}^{\boldsymbol{F}, \boldsymbol{E}}(D):=\rho_{\boldsymbol{M}}^{\boldsymbol{F}, \boldsymbol{E}} \text { (sum of all ways of gluing some legs of } D \text { with } \vartheta_{M}^{\boldsymbol{E}} \text { ). }
$$

Observe that $\kappa_{M}^{\boldsymbol{E}, \boldsymbol{E}}$ is the identity of $\mathcal{A}^{Y}(\boldsymbol{E})$ since $\vartheta_{M}^{\boldsymbol{E}}(\boldsymbol{E}, \boldsymbol{E})=0$ by Lemma A.2. It follows from the next lemma that $\kappa_{M}^{\boldsymbol{F}, \boldsymbol{E}}$ is an isomorphism for any $\boldsymbol{E}, \boldsymbol{F}$.

Lemma A.4 Let $\boldsymbol{E}, \boldsymbol{F}, \boldsymbol{G}$ be essential isotropic subspaces of $H_{1}(\partial M ; \mathbb{Q})$. Then we have $\kappa_{M}^{\boldsymbol{F}, \boldsymbol{E}} \circ \kappa_{M}^{\boldsymbol{G}, \boldsymbol{F}}=\kappa_{M}^{\boldsymbol{G}, \boldsymbol{E}}$.

Proof We need the following identity which is a direct consequence of Lemma A.3:

$$
\vartheta_{\boldsymbol{M}}^{\boldsymbol{E}}(x, y)=\vartheta_{\boldsymbol{M}}^{\boldsymbol{F}}(x, y)+\vartheta_{\boldsymbol{M}}^{\boldsymbol{E}}\left(f g^{-1}(x), f g^{-1}(y)\right) \text { for all } x, y \in \boldsymbol{G} .
$$

Let $D$ be a $\boldsymbol{G}$-colored Jacobi diagram and consider $k$ pairs $\left\{v_{1}, w_{1}\right\}, \ldots,\left\{v_{k}, w_{k}\right\}$ of distinct univalent vertices of $D$ such that $\left\{v_{i}, w_{i}\right\} \cap\left\{v_{j}, w_{j}\right\}=\varnothing$ for any $i \neq j$. By making the identification $v_{i} \equiv w_{i}$ for every $i \in\{1, \ldots, k\}$ and by applying the isomorphism $e^{-1}$ to the colors of the remaining univalent vertices, we obtain an $\boldsymbol{E}$-colored Jacobi diagram $D^{\prime}$. The coefficient of $D^{\prime}$ in the definition of $\kappa_{M}^{\boldsymbol{G}, \boldsymbol{E}}(D)$ is

$$
\begin{aligned}
& \prod_{j=1}^{k} \vartheta_{M}^{\boldsymbol{E}}\left(\operatorname{color}\left(v_{j}\right), \operatorname{color}\left(w_{j}\right)\right) \\
& \stackrel{(\mathrm{A}-6)}{=} \prod_{j=1}^{k}\left(\vartheta_{M}^{\boldsymbol{F}}\left(\operatorname{color}\left(v_{j}\right), \operatorname{color}\left(w_{j}\right)\right)+\vartheta_{M}^{\boldsymbol{E}}\left(f g^{-1} \operatorname{color}\left(v_{j}\right), f g^{-1} \operatorname{color}\left(w_{j}\right)\right)\right) \\
& \quad=\sum_{P \subset\{1, \ldots, k\}} \prod_{p \in P} \vartheta_{M}^{\boldsymbol{F}}\left(\operatorname{color}\left(v_{p}\right), \operatorname{color}\left(w_{p}\right)\right) \\
& \cdot \prod_{q \notin P} \vartheta_{M}^{\boldsymbol{E}}\left(f g^{-1} \operatorname{color}\left(v_{q}\right), f g^{-1} \operatorname{color}\left(w_{q}\right)\right),
\end{aligned}
$$

which is also the coefficient of $D^{\prime}$ in $\kappa_{M}^{\boldsymbol{F}, \boldsymbol{E}}\left(\kappa_{M}^{\boldsymbol{G}, \boldsymbol{F}}(D)\right)$.

\section{A.4 $\mathbb{Q}$-LP surgery equivalence}

We now show that the previous constructions relative to a $\mathbb{Q}$-homology handlebody $M$ only depend on the $\mathbb{Q}$-LP surgery equivalence class of $M$. 
Lemma A.5 Let $M$ be a $\mathbb{Q}$-homology handlebody, let $C=\left(C^{\prime}, C^{\prime \prime}\right)$ be a $\mathbb{Q}$-LP pair such that $C^{\prime} \subset \operatorname{int}(M)$ and let $M_{C}$ be the result of the $\mathbb{Q}-L P$ surgery. Then there is a unique isomorphism $\psi: H_{1}(M ; \mathbb{Q}) \rightarrow H_{1}\left(M_{\mathrm{C}} ; \mathbb{Q}\right)$ such that the following diagram is commutative:

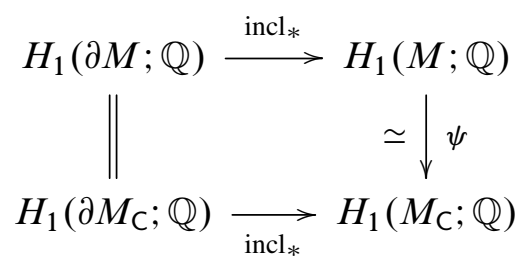

Moreover, any subspace $\boldsymbol{E} \subset H_{1}(\partial M ; \mathbb{Q})$ essential for $M$ is also essential for $M_{\mathrm{C}}$ and $\operatorname{Lk}_{M}^{\boldsymbol{E}}(K, L)=\mathrm{Lk}_{M_{\mathrm{C}}}^{\boldsymbol{E}}(K, L)$ for any disjoint, oriented knots $K, L \subset M \backslash \operatorname{int}\left(C^{\prime}\right)$.

Proof Unicity of $\psi$ follows from the surjectivity of $\operatorname{incl}_{*}: H_{1}(\partial M ; \mathbb{Q}) \rightarrow H_{1}(M ; \mathbb{Q})$. The existence is an application of the Mayer-Vietoris theorem showing that there is a unique isomorphism $\psi: H_{1}(M ; \mathbb{Q}) \rightarrow H_{1}\left(M_{\mathrm{C}} ; \mathbb{Q}\right)$ making the following diagram commutative:

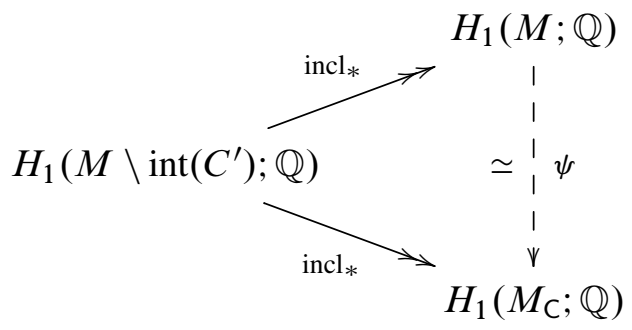

Assume that $\boldsymbol{E} \subset H_{1}(\partial M ; \mathbb{Q})$ is essential for $M$. Since $\psi$ is an isomorphism, $\left.\operatorname{incl}_{*}\right|_{\boldsymbol{E}}=\left.\psi \circ \operatorname{incl}_{*}\right|_{\boldsymbol{E}}: \boldsymbol{E} \rightarrow H_{1}\left(M_{\mathrm{C}} ; \mathbb{Q}\right)$ is an isomorphism so that $\boldsymbol{E}$ is essential for $M_{C}$ as well. Let $K, L \subset M \backslash \operatorname{int}\left(C^{\prime}\right)$ be any two disjoint oriented knots. Let $D$ be a rational 2-chain in $M$ transversal to $K$ such that $\widetilde{L}:=\partial D-L$ is a $1-$ cycle in $\partial M$ and $[\tilde{L}] \in H_{1}(\partial M ; \mathbb{Q})$ belongs to $\boldsymbol{E}$. We can assume that $D=D_{0}+D^{\prime}$, where $D_{0}$ is a rational 2-chain in $M \backslash \operatorname{int}\left(C^{\prime}\right), D^{\prime}$ is a rational 2-chain in $C^{\prime}$ and $\partial D^{\prime}$ is a rational 1-cycle in $\partial C^{\prime}$. Since $\boldsymbol{L}_{C^{\prime}}^{\mathbb{Q}}=\boldsymbol{L}_{C^{\prime \prime}}^{\mathbb{Q}}$, there is a rational 2-chain $D^{\prime \prime}$ in $C^{\prime \prime}$ such that $\partial D^{\prime \prime}=\partial D^{\prime}$. Then $D_{\mathrm{C}}:=D_{0}+D^{\prime \prime}$ is a rational 2-chain in $M_{\mathrm{C}}$ with boundary $\widetilde{L}+L$ and which is transversal to $K$. We conclude thanks to (A-2) that

$$
\operatorname{Lk}_{M}^{E}(K, L)=K \bullet_{M} D=K \bullet_{M_{\mathrm{C}}} D_{\mathrm{C}}=\operatorname{Lk}_{M_{\mathrm{C}}}^{\boldsymbol{E}}(K, L) .
$$

We now fix a closed connected oriented surface $F$. Given a $\mathbb{Q}$-homology handlebody $M$ with boundary parameterization $m: F \rightarrow M$, a subspace $\boldsymbol{E}$ of $H_{1}(F ; \mathbb{Q})$ is said to be $M$-essential if $m_{*}(\boldsymbol{E})$ is essential in the sense of Section A.1, ie the restriction to $\boldsymbol{E}$ of $m_{*}: H_{1}(F ; \mathbb{Q}) \rightarrow H_{1}(M ; \mathbb{Q})$ is an isomorphism onto $H_{1}(M ; \mathbb{Q})$. 
Lemma A.6 Let $(M, m)$ and $(\bar{M}, \bar{m})$ be $\mathbb{Q}$-homology handlebodies whose boundaries are parameterized by $F$. Then the following statements are equivalent:

(i) $(\bar{M}, \bar{m})$ can be obtained from $(M, m)$ by a single $\mathbb{Q}-L P$ surgery.

(i') $(\bar{M}, \bar{m})$ can be obtained from $(M, m)$ by a sequence of $\mathbb{Q}$-LP surgeries.

(ii) We have $m_{*}^{-1}\left(\boldsymbol{L}_{M}^{\mathbb{Q}}\right)=\bar{m}_{*}^{-1}\left(\boldsymbol{L} \frac{\mathbb{Q}}{M}\right)$ in $H_{1}(F ; \mathbb{Q})$.

(iii) There is a subspace $\boldsymbol{E} \subset H_{1}(F ; \mathbb{Q})$ which is $M$-and $\bar{M}$-essential, such that

$$
\vartheta_{\boldsymbol{M}}^{m_{*}(\boldsymbol{E})} \circ\left(m_{*} \times m_{*}\right)=\vartheta_{\overline{\boldsymbol{M}}}^{\bar{m}_{*}(\boldsymbol{E})} \circ\left(\bar{m}_{*} \times \bar{m}_{*}\right) .
$$

Proof Clearly (i) implies $\left(i^{\prime}\right)$. Assume $\left(i^{\prime}\right)$ : there is a sequence of $\mathbb{Q}-$ LP surgeries

$$
(M, m)=\left(M_{1}, m_{1}\right) \rightsquigarrow\left(M_{2}, m_{2}\right) \rightsquigarrow \cdots \rightsquigarrow\left(M_{r+1}, m_{r+1}\right)=(\bar{M}, \bar{m}) .
$$

For all $i \in\{1, \ldots, r\}$, the isomorphism $\psi_{i}: H_{1}\left(M_{i} ; \mathbb{Q}\right) \rightarrow H_{1}\left(M_{i+1} ; \mathbb{Q}\right)$ given by Lemma A.5 satisfies $\psi_{i} \circ m_{i, *}=m_{i+1, *}: H_{1}(F ; \mathbb{Q}) \rightarrow H_{1}\left(M_{i+1} ; \mathbb{Q}\right)$. Hence the composite isomorphism $\psi:=\psi_{r} \circ \cdots \circ \psi_{1}$ satisfies $\psi \circ m_{*}=\bar{m}_{*}$. For any $x \in m_{*}^{-1}\left(\boldsymbol{L}_{M}^{\mathbb{Q}}\right)$, we have

$$
\operatorname{incl}_{*} \bar{m}_{*}(x)=\bar{m}_{*}(x)=\psi m_{*}(x)=\psi \operatorname{incl}_{*} m_{*}(x)=0,
$$

so that $x \in \bar{m}_{*}^{-1}\left(\boldsymbol{L} \frac{\mathbb{Q}}{M}\right)$. This shows that

$$
m_{*}^{-1}\left(\boldsymbol{L}_{M}^{\mathbb{Q}}\right) \subset \bar{m}_{*}^{-1}\left(\boldsymbol{L} \frac{\mathbb{Q}}{M}\right)
$$

and the opposite inclusion is proved similarly: hence we get (ii). Clearly, (ii) implies (i) and it now remains to prove the equivalence between (iii) and (i), (i') and (ii).

That (i) implies (iii) follows from the second statement of Lemma A.5. Assume (iii) and denote by $h: \partial M \rightarrow \partial \bar{M}$ the orientation-preserving homeomorphism defined by $h(m(x))=\bar{m}(x)$ for any $x \in F$. Then we have

$$
\vartheta_{M}^{m_{*}(\boldsymbol{E})}=\vartheta_{\bar{M}}^{\bar{m}_{*}(\boldsymbol{E})} \circ\left(h_{*} \times h_{*}\right)
$$

so that $h_{*}$ sends the left radical of $\vartheta_{M}^{m_{*}(\boldsymbol{E})}$ to the left radical of $\vartheta_{\bar{M}}^{\bar{m}_{*}(\boldsymbol{E})}$. We deduce assertion (ii) using the second statement of Lemma A.2.

Fix a $\mathbb{Q}$-LP surgery equivalence class $\mathcal{M}$ of $\mathbb{Q}$-homology handlebodies with boundary parameterized by $F$. By the equivalence (i) $\Leftrightarrow$ (ii) in Lemma A.6, the choice of $\mathcal{M}$ is equivalent to the choice of a Lagrangian subspace $\boldsymbol{L}$ of $H_{1}(F ; \mathbb{Q})$, satisfying $m_{*}(\boldsymbol{L})=\boldsymbol{L}_{M}^{\mathbb{Q}}$ for any $M \in \mathcal{M}$. A subspace $\boldsymbol{E}$ of $H_{1}(F ; \mathbb{Q})$ is said to be $\mathcal{M}-$ essential if $H_{1}(F ; \mathbb{Q})=\boldsymbol{L} \oplus \boldsymbol{E}$ and, in this case, it induces a bilinear form

$$
\vartheta_{\mathcal{M}}^{\boldsymbol{E}}: H_{1}(F ; \mathbb{Q}) \times H_{1}(F ; \mathbb{Q}) \longrightarrow \mathbb{Q}
$$


defined by $\vartheta_{\mathcal{M}}^{\boldsymbol{E}}\left(l_{1}+e_{1}, l_{2}+e_{2}\right):=l_{2} \bullet_{F} e_{1}$ for any $l_{1}, l_{2} \in \boldsymbol{L}$ and $e_{1}, e_{2} \in \boldsymbol{E}$. Observe that $\boldsymbol{E}$ is $\mathcal{M}$-essential if and only if it is $M$-essential for a particular $M \in \mathcal{M}$ and, by Lemma A.2, $\vartheta_{\mathcal{M}}^{\boldsymbol{E}}$ then corresponds to $\vartheta_{M}^{m_{*}(\boldsymbol{E})}$ through the isomorphism $m_{*}: H_{1}(F ; \mathbb{Q}) \rightarrow H_{1}(\partial M ; \mathbb{Q})$.

Furthermore, for any two isotropic $\mathcal{M}$-essential subspaces $\boldsymbol{E}, \boldsymbol{F} \subset H_{1}(F ; \mathbb{Q})$, there is a linear map

$$
\kappa_{\mathcal{M}}^{\boldsymbol{F}, \boldsymbol{E}}: \mathcal{A}^{Y}(\boldsymbol{F}) \longrightarrow \mathcal{A}^{Y}(\boldsymbol{E})
$$

defined for any $\boldsymbol{F}$-colored Jacobi diagram $D$ by

$$
\kappa_{\mathcal{M}}^{\boldsymbol{F}, \boldsymbol{E}}(D):=\rho_{\mathcal{M}}^{\boldsymbol{F}, \boldsymbol{E}} \text { (sum of all ways of gluing some legs of } D \text { with } \vartheta_{\mathcal{M}}^{\boldsymbol{E}} \text { ), }
$$

where $\rho_{\mathcal{M}}^{\boldsymbol{F}, \boldsymbol{E}}: \mathcal{A}^{Y}(\boldsymbol{F}) \rightarrow \mathcal{A}^{Y}(\boldsymbol{E})$ is the isomorphism induced by the change of colors

$$
\boldsymbol{F} \stackrel{\text { inclusion }}{\longrightarrow} \boldsymbol{L} \oplus \boldsymbol{F}=H_{1}(F ; \mathbb{Q})=\boldsymbol{L} \oplus \boldsymbol{E} \stackrel{\text { projection }}{\longrightarrow} \boldsymbol{E} .
$$

Observe that the map $\kappa_{\mathcal{M}}^{\boldsymbol{F}, \boldsymbol{E}}$ corresponds to the isomorphism $\kappa_{M}^{m_{*}(\boldsymbol{F}), m_{*}(\boldsymbol{E})}$ for any $M \in \mathcal{M}$ through the isomorphisms

$$
\mathcal{A}^{Y}(\boldsymbol{E}) \simeq \mathcal{A}^{Y}\left(m_{*}(\boldsymbol{E})\right) \text { and } \quad \mathcal{A}^{Y}(\boldsymbol{F}) \simeq \mathcal{A}^{Y}\left(m_{*}(\boldsymbol{F})\right)
$$

that are induced by the changes of colors $\left.m_{*}\right|_{\boldsymbol{E}}: \boldsymbol{E} \rightarrow m_{*}(\boldsymbol{E}) \subset H_{1}(\partial M ; \mathbb{Q})$ and $\left.m_{*}\right|_{\boldsymbol{F}}: \boldsymbol{F} \rightarrow m_{*}(\boldsymbol{F}) \subset H_{1}(\partial M ; \mathbb{Q})$, respectively.

\section{Appendix B: Milnor's triple linking numbers in $\mathbb{Q}$-homology 3-spheres}

We extend the definition of Milnor's triple linking numbers in $S^{3}$ to any $\mathbb{Q}$-homology 3 -sphere and we relate them to the Kontsevich-LMO invariant. This relation is needed in the proof of the splitting formulas.

\section{B.1 Definition}

Let $L$ be an algebraically-split oriented link in a $\mathbb{Q}$-homology 3-sphere $S$, whose connected components are numbered from 1 to $\ell$ :

$$
\operatorname{Lk}_{S}\left(L_{i}, L_{j}\right)=0 \quad \text { for all } i \neq j \in\{1, \ldots, \ell\} .
$$

Then, for each triplet $(i, j, k)$ of distinct integers in $\{1, \ldots, \ell\}$, there is an isotopy invariant of the link $L$ in $S$

$$
\bar{\mu}_{i j k}(L) \in \mathbb{Q},
$$


which, in the case of $S=S^{3}$, is the usual Milnor's isotopy invariant of length 3 . In general, $\bar{\mu}_{i j k}(L)$ is not an integer but a rational number.

The most efficient, although indirect, way to define $\bar{\mu}_{i j k}(L)$ is as follows. Let $S_{L}$ be the closed oriented 3-manifold obtained from $S$ by longitudinal surgery along $L$. Thus $S_{L}$ is obtained from the exterior $S \backslash \operatorname{int}(N(L))$ of the link $L$ by gluing $\ell$ solid tori, the meridian of the $i^{\text {th }}$ solid torus being glued to the longitude ${ }^{1} \lambda_{i}$ of the $i^{\text {th }}$ component of $L$. The vector space $H_{1}\left(S_{L} ; \mathbb{Q}\right)$ has dimension $\ell$, with preferred basis given by the images of the oriented meridians $m_{1}, \ldots, m_{\ell}$ of $L_{1}, \ldots, L_{\ell}$ under $\operatorname{incl}_{*}: H_{1}(S \backslash \operatorname{int}(N(L)) ; \mathbb{Q}) \rightarrow H_{1}\left(S_{L} ; \mathbb{Q}\right)$. Let also $\left(m_{1}^{*}, \ldots, m_{\ell}^{*}\right)$ be the dual basis of $H^{1}\left(S_{L} ; \mathbb{Q}\right)$, ie $\left\langle m_{i}^{*}, m_{j}\right\rangle=\delta_{i, j}$ for all $i, j=1, \ldots, \ell$. Then

$$
\bar{\mu}_{i j k}(L):=\left\langle m_{i}^{*} \cup m_{j}^{*} \cup m_{k}^{*},\left[S_{L}\right]\right\rangle \in \mathbb{Q}
$$

defines an isotopy invariant of $L \subset S$.

The invariant $\bar{\mu}_{i j k}(L)$ can be computed as follows. Since the link $L$ is assumed to be algebraically-split, each component $L_{i}$ of $L$ is rationally nullhomologous in the exterior of the other components. Thus we can find some compact connected oriented surfaces $\Sigma_{1}, \ldots, \Sigma_{\ell} \subset S$ satisfying the following:

- For all $i \in\{1, \ldots, \ell\}$, there exists an integer $n_{i}>0$ such that $\partial \Sigma_{i}$ winds $n_{i}$ times around $L_{i}$.

- For all $i \neq j \in\{1, \ldots, \ell\}, \Sigma_{i}$ is in transverse position with $\Sigma_{j}$ and $\Sigma_{i} \cap L_{j}=\varnothing$.

Lemma B.1 With the above notation, we have

$$
\bar{\mu}_{i j k}(L)=-\frac{\Sigma_{i} \cdot \Sigma_{j} \cdot \Sigma_{k}}{n_{i} n_{j} n_{k}} \in \mathbb{Q} .
$$

In this form, the invariant $\bar{\mu}_{i j k}(L)$ appears in [19]. Here the intersection number $\Sigma_{i} \cdot \Sigma_{j} \cdot \Sigma_{k}$ in $S$ is computed with the sign convention of Bredon [5] (which agrees with that of [19]).

Proof of Lemma B.1 We can assume after an isotopy that, for each $i \in\{1, \ldots, \ell\}$, the surface $\Sigma_{i}$ does not cut $N\left(L_{j}\right)$ for $j \neq i$ and is in transverse position with $\partial N\left(L_{i}\right)$. Thus $\Sigma_{i} \cap \partial N\left(L_{i}\right)$ consists of nullhomotopic simple closed curves in $\partial N\left(L_{i}\right)$ and a family of simple closed curves parallel to the same curve. Since this curve is then rationally nullhomologous in the complement of $N\left(L_{i}\right)$, it is necessarily

${ }^{1}$ The longitude of an oriented knot $K$ in a $\mathbb{Q}$-homology 3-sphere $S$ is the unique oriented simple closed curve on $\partial N(K)$ that is rationally nullhomologous in $S \backslash \operatorname{int}(N(K))$ and that is homotopic in $N(K)$ to $b \cdot K$ for some integer $b>0$. This is not necessarily a parallel of $K$, ie we may have $b>1$. 
the longitude $\lambda_{i}$ of $L_{i}$ : let $r_{i} \in \mathbb{Z}$ be the number of times (counted with signs) $\Sigma_{i} \cap(S \backslash \operatorname{int}(N(L)))$ winds around $\lambda_{i}$. Because the surgery $S \rightsquigarrow S_{L}$ is longitudinal, we can transform $\Sigma_{i} \cap(S \backslash \operatorname{int}(N(L)))$ to a closed oriented surface $\left(\Sigma_{i}\right)_{L} \subset S_{L}$ by gluing some disks inside the $i^{\text {th }}$ surgery solid torus. Let $p_{i} \subset \partial N\left(L_{i}\right)$ be an oriented parallel of $L_{i}$, and let $a_{i}, b_{i}$ be the integers satisfying $\lambda_{i}=a_{i} \cdot m_{i}+b_{i} \cdot p_{i} \in H_{1}\left(\partial N\left(L_{i}\right) ; \mathbb{Z}\right)$. We have $\left(\Sigma_{i}\right)_{L} \cap m_{j}=\varnothing$ for all $j \neq i$ and

$$
m_{i} \cdot\left(\Sigma_{i}\right)_{L}=m_{i} \cdot\left(r_{i} \lambda_{i}\right)=r_{i} b_{i}=n_{i} .
$$

Therefore, $\left[\frac{1}{n_{i}}\left(\Sigma_{i}\right)_{L}\right] \in H_{2}\left(S_{L} ; \mathbb{Q}\right)$ is Poincaré dual to $m_{i}^{*} \in H^{1}\left(S_{L} ; \mathbb{Q}\right)$. Thanks to the Poincaré correspondence between homological intersections and cup products (using the conventions of [5]), we have

$$
\begin{aligned}
\bar{\mu}_{i j k}(L) & =\left\langle m_{i}^{*} \cup m_{j}^{*} \cup m_{k}^{*},\left[S_{L}\right]\right\rangle \\
& =-\left[\frac{1}{n_{i}}\left(\Sigma_{i}\right)_{L}\right] \cdot\left[\frac{1}{n_{j}}\left(\Sigma_{j}\right)_{L}\right] \cdot\left[\frac{1}{n_{k}}\left(\Sigma_{k}\right)_{L}\right]=-\frac{\Sigma_{i} \cdot \Sigma_{j} \cdot \Sigma_{k}}{n_{i} n_{j} n_{k}} .
\end{aligned}
$$

This concludes the proof.

\section{B.2 The "Y" part of the Kontsevich-LMO invariant}

The relationship between $\bar{\mu}$-invariants of links in $S^{3}$ and the Kontsevich integral has been studied by Habegger and Masbaum who considered $\mu$-invariants of string-links in the standard cube [9]. (See also [22] for the case of string-links in $\mathbb{Z}$-homology cubes.) The following deals with the length three $\bar{\mu}$-invariants of algebraically-split links in $\mathbb{Q}$-homology 3-spheres, as defined in Section B.1.

Lemma B.2 Let $(B, \gamma)$ be an $\ell$-component top tangle in a $\mathbb{Q}$-homology cube, and assume that the plat closure $\hat{\gamma}$ of $\gamma$ in the $\mathbb{Q}$-homology 3-sphere $\hat{B}$ is algebraicallysplit. Then the Kontsevich-LMO invariant of $(B, \gamma)$ determines the framing numbers ${ }^{2}$ of $\hat{\gamma}$ and its Milnor's triple linking numbers as follows:

$\log _{\sqcup} \chi^{-1} Z(B, \gamma)=\sum_{i=1}^{\ell} \frac{\operatorname{Fr}\left(\hat{\gamma}_{i}\right)}{2} i^{+} i^{+}+\sum_{1 \leq i<j<k \leq \ell} \bar{\mu}_{i j k}(\hat{\gamma}) k^{+} j^{+} i^{+}+(\mathrm{i}-\operatorname{deg}>1)$.

Here the Kontsevich-LMO invariant $Z(B, \gamma)$ of the top tangle $(B, \gamma)$ is as defined in [6, Section 3.5] (and as briefly recalled in Section 2). The top tangle $\gamma$ is equipped with any nonassociative word of length $\ell$ in the single letter $\bullet$ to which the rule $\bullet \mapsto(+-)$ is applied.

2 The framing number $\operatorname{Fr}(K) \in \mathbb{Q}$ of a framed knot $K$ in a $\mathbb{Q}$-homology 3 -sphere $S$ is the linking number in $S$ of $K$ with the parallel of $K$ defined by the framing. 
Proof of Lemma B.2 The fact that $\chi^{-1} Z(B, \gamma) \in \mathcal{A}\left(\lfloor\ell\rceil^{+}\right)$is of the form

$$
\exp _{\sqcup}\left(\sum_{i=1}^{\ell} \frac{\operatorname{Fr}\left(\hat{\gamma}_{i}\right)}{2}{\stackrel{i}{+\ldots} i^{+}}^{+}+\sum_{1 \leq i<j<k \leq \ell} a_{i j k}(\gamma) \cdot k^{+} j^{+} i^{+}+(\mathrm{i}-\operatorname{deg}>1)\right)
$$

for some $a_{i j k}(\gamma) \in \mathbb{Q}$ is well known (see [6, Lemma 3.17] for example). In the sequel, we denote by $z(B, \gamma)$ the "Y" part of $\chi^{-1} Z(B, \gamma)$ and by $m(B, \gamma)$ the linear combination of $Y$-shaped diagrams defined by Milnor's triple linking numbers of $\hat{\gamma}$ in $\widehat{B}$ :

$$
z(B, \gamma):=\sum_{1 \leq i<j<k \leq \ell} a_{i j k}(\gamma) \cdot{ }^{k+j^{+} i^{+}}, \quad m(B, \gamma):=\sum_{1 \leq i<j<k \leq \ell} \bar{\mu}_{i j k}(\hat{\gamma}) \cdot k^{+} j^{+} i^{+}
$$

We are asked to prove that $m(B, \gamma)=-z(B, \gamma)$.

For any integer $r \geq 1$ and for any $i \in\{1, \ldots, \ell\}$, let $\gamma_{r \times i}$ be a top tangle in $B$ which is identical to $\gamma$, except that the $i^{\text {th }}$ component of $\gamma_{r \times i}$ now goes $r$ times around the $i^{\text {th }}$ component of $\gamma$. Then it can be deduced from the "doubling property" of the Kontsevich integral that $z\left(B, \gamma_{r \times i}\right)$ is obtained from $z(B, \gamma)$ by the operation $i^{+} \mapsto r \cdot i^{+}$. (Note that the version of the Kontsevich integral used in [6] has a "doubling anomaly", but this does not affect $z(B, \gamma)$.) Besides, it follows from Lemma B.1 that $m\left(B, \gamma_{r \times i}\right)$ differs from $m(B, \gamma)$ in the same way. Therefore we can assume in the sequel that each component of $\hat{\gamma}$ is nullhomologous in $\widehat{B}$ (with coefficients in $\mathbb{Z}$ ).

For any $n \in \mathbb{Z}$ and for any $i \in\{1, \ldots, \ell\}$, let $\gamma_{n \rightarrow i}$ be the top tangle in $B$ that is obtained from $\gamma$ by changing the framing of $\gamma_{i}$ by $n$ (ie by adding $|n|$ "kinks" of the same sign as $n$ to $\gamma$ ). This operation modifies the Kontsevich integral by the exponential of an isolated chord (times $n / 2)$ so that we have $z(B, \gamma)=z\left(B, \gamma_{n} \rightarrow i\right)$. Since Milnor's triple linking numbers of $\hat{\gamma}$ do not depend on the framing of $\hat{\gamma}$, we also have $m(B, \gamma)=m\left(B, \gamma_{n \rightarrow i}\right)$. So we can assume in the sequel that the framing number of each component of $\hat{\gamma}$ is zero.

With the above two assumptions on $\hat{\gamma}$, we can find for each $i \in\{1, \ldots, \ell\}$ a Seifert surface $\Sigma_{i}$ for the framed knot $\hat{\gamma}_{i}$ such that $\Sigma_{i}$ is in transverse position with $\Sigma_{j}$ and does not meet $\hat{\gamma}_{j}$ for all $j \neq i$. The standard cube $[-1,1]^{3}$ can be obtained from $B$ by surgery along a framed link $L^{*}$ and, by an isotopy of the link $L^{*}$ in $B$, we can require that $L^{*} \subset \widehat{B}$ is disjoint from $\hat{\gamma}$ and that each component of $L^{*}$ has a trivial linking number with every component of $\hat{\gamma}$. Thus, by adding "tubes" to the Seifert surfaces $\Sigma_{1}, \ldots, \Sigma_{\ell}$, we can assume that each of them is disjoint from $L^{*}$. Then the framed link $L \subset[-1,1]^{3}$ dual to $L^{*}$ has the following two properties: first, surgery along $L$ produces $B$; second, the surfaces $\Sigma_{1}, \ldots, \Sigma_{\ell}$ (and, a fortiori, their boundaries $\left.\widehat{\gamma}_{1}, \ldots, \hat{\gamma}_{\ell}\right)$ can be seen in the exterior $[-1,1]^{3} \backslash \operatorname{int}(N(L))$. Therefore, the pair $(L, \gamma)$ 
is a surgery presentation of $(B, \gamma)$ in $[-1,1]^{3}$ satisfying $\operatorname{Lk}_{[-1,1]^{3}}(L, \gamma)=0$ and $\operatorname{Lk}_{[-1,1]^{3}}(\gamma)=0$. Then, using the formal Gaussian integration of [2;3] and adopting the notation of [6, Section 3], we have

$$
\begin{aligned}
z(B, \gamma) & =\text { "Y" part of } U_{+}^{-\sigma_{+}(L)} \sqcup U_{-}^{-\sigma_{-}(L)} \sqcup \int_{\pi_{0}(L)} \chi^{-1} Z\left(L^{\nu} \cup \gamma\right) \\
& =\text { "Y" part of } \int_{\pi_{0}(L)} \chi^{-1} Z\left(L^{\nu} \cup \gamma\right)=\text { "Y" part of } \int_{\pi_{0}(L)} \chi^{-1} Z(L \cup \gamma),
\end{aligned}
$$

where the last identity follows from the fact that $\chi^{-1}$ preserves the i-degree filtration. Next, we have $\chi^{-1} Z(L \cup \gamma)=\exp _{\sqcup}(A / 2) \sqcup \exp _{\sqcup}(T)$ where the matrix $A:=\mathrm{Lk}_{[-1,1]^{3}}(L)$ is regarded as a linear combination of struts and where $T$ consists of connected Jacobi diagrams colored by $\pi_{0}(L \cup \gamma)$ of i-degree greater than or equal to 1 . So we have

$$
\int_{\pi_{0}(L)} \chi^{-1} Z(L \cup \gamma)=\left\langle\exp _{\sqcup}\left(-A^{-1} / 2\right), \exp _{\sqcup}(T)\right\rangle_{\pi_{0}(L)}
$$

and we deduce that $z(B, \gamma)$ is the "Y" part of $\chi^{-1} Z(L \cup \gamma)$ that only involves $\pi_{0}(\gamma)$, ie the "Y" part of $\chi^{-1} Z(\gamma)$. Besides, we deduce from Lemma B.1 that the Milnor invariant $\bar{\mu}_{i j k}(\hat{\gamma})$ for $\hat{\gamma} \subset \widehat{B}$ is the same as for $\hat{\gamma} \subset \overline{[-1,1]^{3}}=S^{3}$. Therefore we can assume in the sequel that $B$ is the standard cube $[-1,1]^{3}$.

With this assumption on $B$, the invariant $Z(B, \gamma)$ is the usual Kontsevich integral $Z(\gamma)$ of the $q$-tangle $\gamma$ (as it is normalized in [6]). Then we can appeal to [9] or, alternatively, we can proceed as follows. If $\gamma$ is the trivial $\ell$-component top tangle, then the "Y" part of $Z(\gamma)$ is trivial and we are done. Next, we assume that $\gamma$ is any top tangle in $B=[-1,1]^{3}$, still with the assumption $\operatorname{Lk}_{B}(\gamma)=0$. Then, according to Matveev [21] and Murakami and Nakanishi [24], $\gamma$ can be obtained from the trivial top tangle by a finite number of "Borromean transformations". Such a transformation performed on the $i^{\text {th }}, j^{\text {th }}$ and $k^{\text {th }}$ components of $\gamma$ produces in $z(B, \gamma)$ a variation by

$$
\begin{array}{ll}
k+j^{+} & i^{+} \\
\varepsilon & \vdots
\end{array}
$$

(see Ohtsuki [25, Lemma 11.22]). Here $\varepsilon= \pm 1$ is a sign that depends on the configuration of the Borromean rings. It is easily checked that the same "Borromean transformation" produces for $\bar{\mu}_{i j k}(\hat{\gamma})$ a variation by $-\varepsilon$.

\section{References}

[1] E Auclair, C Lescop, Clover calculus for homology 3-spheres via basic algebraic topology, Algebr. Geom. Topol. 5 (2005) 71-106 MR2135546 
[2] D Bar-Natan, S Garoufalidis, L Rozansky, DP Thurston, The Århus integral of rational homology 3-spheres, I: A highly nontrivial flat connection on $S^{3}$, Selecta Math. 8 (2002) 315-339 MR1931167

[3] D Bar-Natan, S Garoufalidis, L Rozansky, D P Thurston, The Århus integral of rational homology 3-spheres, II: Invariance and universality, Selecta Math. 8 (2002) 341-371 MR1931168

[4] D Bar-Natan, S Garoufalidis, L Rozansky, DP Thurston, The Arhus integral of rational homology 3-spheres, III: Relation with the Le-Murakami-Ohtsuki invariant, Selecta Math. 10 (2004) 305-324 MR2099069

[5] GE Bredon, Topology and geometry, Graduate Texts in Math. 139, Springer, New York (1993) MR1224675

[6] D Cheptea, K Habiro, G Massuyeau, A functorial LMO invariant for Lagrangian cobordisms, Geom. Topol. 12 (2008) 1091-1170 MR2403806

[7] D Cimasoni, V Turaev, A generalization of several classical invariants of links, Osaka J. Math. 44 (2007) 531-561 MR2360939

[8] L Crane, D Yetter, On algebraic structures implicit in topological quantum field theories, J. Knot Theory Ramifications 8 (1999) 125-163 MR1687553

[9] N Habegger, G Masbaum, The Kontsevich integral and Milnor's invariants, Topology 39 (2000) 1253-1289 MR1783857

[10] K Habiro, Claspers and finite type invariants of links, Geom. Topol. 4 (2000) 1-83 MR1735632

[11] K Habiro, G Massuyeau, Symplectic Jacobi diagrams and the Lie algebra of homology cylinders, J. Topol. 2 (2009) 527-569 MR2546585

[12] T Kerler, Towards an algebraic characterization of 3-dimensional cobordisms, from: "Diagrammatic morphisms and applications", (D E Radford, F J O Souza, D N Yetter, editors), Contemp. Math. 318, Amer. Math. Soc. (2003) 141-173 MR1973515

[13] T T Q Le, An invariant of integral homology 3-spheres which is universal for all finite type invariants, from: "Solitons, geometry, and topology: on the crossroad", (V M Buchstaber, S P Novikov, editors), Amer. Math. Soc. Transl. Ser. 2 179, Amer. Math. Soc. (1997) 75-100 MR1437158

[14] T T Q Le, J Murakami, Representation of the category of tangles by Kontsevich's iterated integral, Comm. Math. Phys. 168 (1995) 535-562 MR1328252

[15] T T Q Le, J Murakami, The universal Vassiliev-Kontsevich invariant for framed oriented links, Compositio Math. 102 (1996) 41-64 MR1394520

[16] T T Q Le, J Murakami, T Ohtsuki, On a universal perturbative invariant of 3manifolds, Topology 37 (1998) 539-574 MR1604883

[17] C Lescop, Splitting formulae for the Kontsevich-Kuperberg-Thurston invariant of rational homology 3-spheres arXiv:math.GT/0411431 
[18] C Lescop, A sum formula for the Casson-Walker invariant, Invent. Math. 133 (1998) 613-681 MR1645066

[19] C Lescop, Surgery formulae for finite type invariants of rational homology 3-spheres, Algebr. Geom. Topol. 9 (2009) 979-1047 MR2511138

[20] G Massuyeau, J-B Meilhan, Equivalence relations for homology cylinders and the core of the Casson invariant, Trans. Amer. Math. Soc. 365 (2013) 5431-5502 MR3074379

[21] S V Matveev, Generalized surgeries of three-dimensional manifolds and representations of homology spheres, Mat. Zametki 42 (1987) 268-278, 345 MR915115

[22] I Moffatt, The Århus integral and the $\mu$-invariants, J. Knot Theory Ramifications 15 (2006) 361-377 MR2217502

[23] D Moussard, Finite type invariants of rational homology 3-spheres, Algebr. Geom. Topol. 12 (2012) 2389-2428 MR3020211

[24] H Murakami, Y Nakanishi, On a certain move generating link-homology, Math. Ann. 284 (1989) 75-89 MR995383

[25] T Ohtsuki, Quantum invariants, Series on Knots and Everything 29, World Scientific, River Edge, NJ (2002) MR1881401

[26] K Walker, An extension of Casson's invariant, Annals of Math. Studies 126, Princeton Univ. Press (1992) MR1154798

Institut de Recherche Mathématique Avancée, Université de Strasbourg and CNRS 7 rue René Descartes, 67084 Strasbourg, France

massuyeau@math.unistra.fr

http://www-irma.u-strasbg.fr/ massuyea/

Received: 11 October 2013 